\title{
THE EFFECTS OF THE GALILEAN MOONS ON JUPITER FLYBY TRAJECTORIES
}

\section{BERNAR'D KAUFMAN}

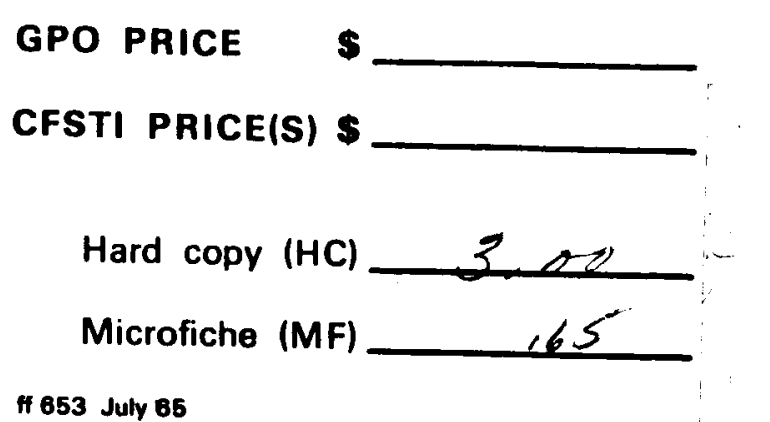

SEPTEMBER 1967

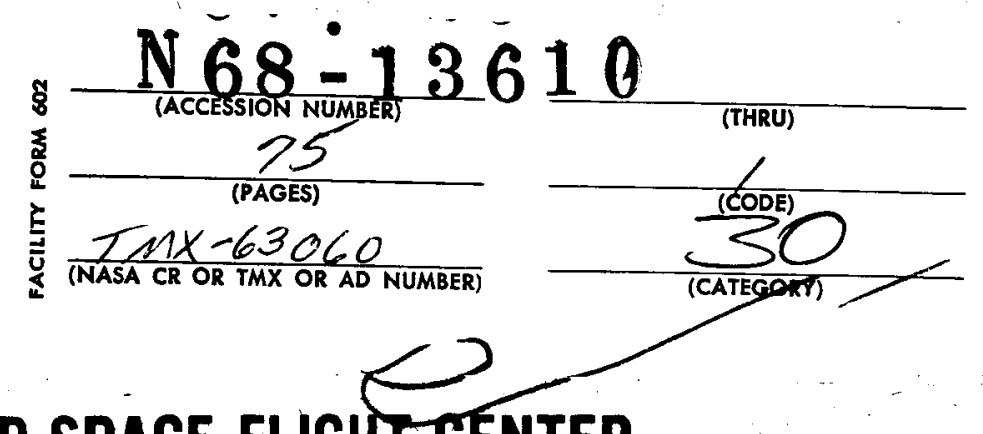

NASA

GODDARD SPACE FLIGHT CENTER

GREENBELT, MARYLAND 


\title{
THE EFFECTS OF THE GALILEAN MOONS ON JUPITER FLYBY TRAJECTORIES
}

\author{
Bernard Kaufman
}

September 1967

\author{
Mission and Systems Analysis Branch \\ Mission and Trajectory Analysis Division \\ Goddard Space Flight Center \\ Greenbelt, Maryland
}




\author{
NOT FILAD \\ THE EFFECTS OF THE GALILEAN MOONS ON \\ JUPITER FLYBY TRAJECTORIES \\ by \\ Bernard Kaufman
}

\begin{abstract}
The perturbative effects of the four largest moons of Jupiter on a spacecraft that passes close to Jupiter are investigated using a total of twenty different encounter trajectories with the planet. An accurate model for the moons is utilized in the equations of motion which are numerically integrated over the time that the probe spends within Jupiter's sphere of influence. It is shown that the perturbations caused by the moons do have effects on the post encounter heliocentric trajectories which are large enough to make necessary the inclusion of the moons in any detailed study to be undertaken. On the other hand, actual mission objectives such as the time to 10 astronomical units seem to be little affected by the presence of the moons.

A valuable by-product of this study has been the programming of an accurate ephemeris for the Galilean moons which has not previously been available in this country as far as the author has been able to determine.
\end{abstract}




\section{ACKNOWLEDGEMENTS}

I would like to thank Dr. Peter Musen for the many hours that he has spent helping me overcome the many problems encountered in this study and for his patience in seeing this project through to the end. I also would like to thank Mr. R. Groves for his understanding and patience in waiting for the results of this study. To Mr. Charles Newman must also go my deepest appreciation for his valuable help in debugging the computer program and his many helpful suggestions. Dr. Jean L. Sagnier of the Bureau des Longitudes in Paris, France spent much of his own time in order to supply me with a simplified method of determining accurate positions of the Galilean moons. Without his help it is doubtful that this study could have been made. 
CONTENTS

$\underline{\text { Page }}$

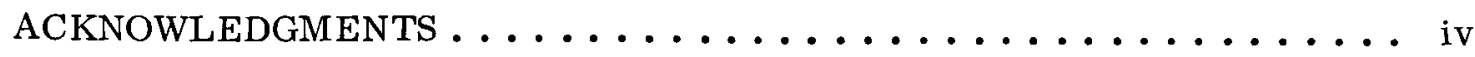

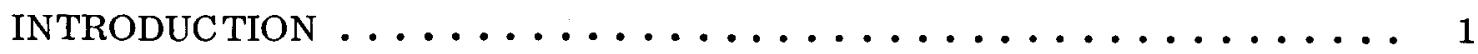

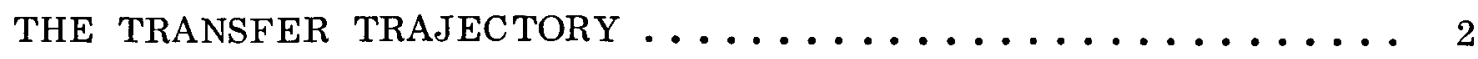

UNDISTURBED JUPITER FLYBY AND POST

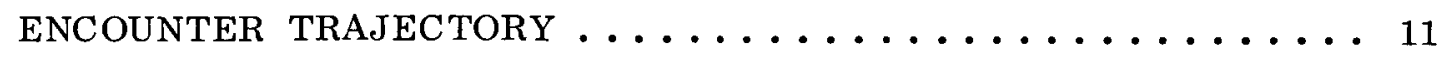

The Sphere of Influence ............................. 11

The Undisturbed Post Encounter Trajectory . . . . . . . . . . . 13

THE PERTURBED JUPITER FLYBY . . . . . . . . . . . . . . 21

Cowell's Equations of Motion-Perturbations in

Rectangular Coordinates ..................... 21

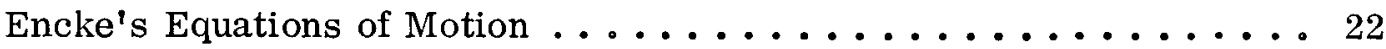

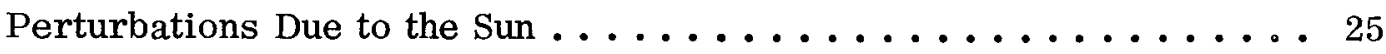

Combining the Equations of Motion ................. 27

Integration of the Equations of Motion . . . . . . . . . . . . 28

THE GALILEAN SATELLITES OF JUPITER . . . . . . . . . . . 30

ENCOUNTER GEOMETRIES AND MISSIONS USED IN THE STUDY . . . 34

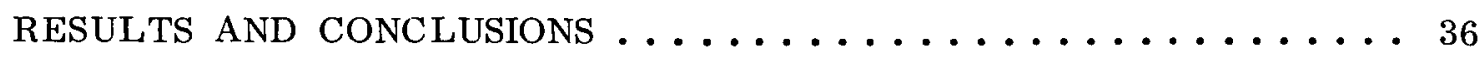

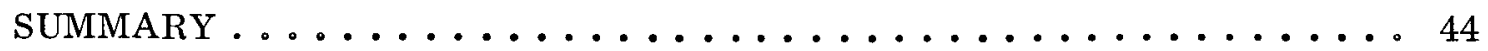

APPENDIX A. TO COMPUTE TIME FROM PERIAPSIS TO A GIVEN

TRUE ANOMALY . . . . . . . . . . . . . . . 4 46

APPENDIX B. TO UPDATE THE CARTESIAN COORDINATES OF

THE PROBE USING AN INCREMENTAL TRUE

ANOMALY ......................... . . . 49

APPENDIX C. TO FIND $\vec{r}$ AND $\vec{v}$ OF A PROBE ON AN HYPERBOLIC ORBIT SEPARATED FROM $\overrightarrow{\mathrm{r}}_{0}$ AND $\overrightarrow{\mathrm{v}}_{0}$ BY

TIME $\Delta t \ldots \ldots \ldots \ldots \ldots \ldots \ldots \ldots$

APPENDIX D. THE POSITIONS OF JUPITER'S GREAT SATELLITES . . 58

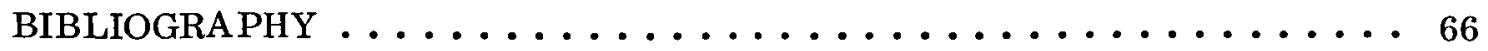




\section{LIST OF TABLES}

Table

Page

I The Velocity Vector as a Function of Choosing the Entrance Point on the Sphere of Influence . . . . . . . . . . . . . . 15

II Effects of Approximating the Encounter Trajectory . . . . . . . 20

III The Galilean Satellites: Astronomical and Physical Data . . . . 31

IV Comparison of Sagnier's method and Sampson's Tables

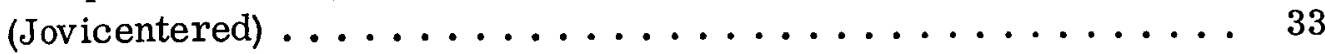

$\mathrm{V}$ Effects of the Galilean Moons on the Jovicentric Orbital Elements ............................... 40

VI Effects of the Galilean Moons on the Heliocentric Orbital

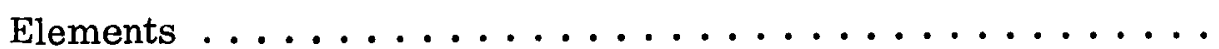

VII Effects of the Galilean Moons on Deep Space Mission

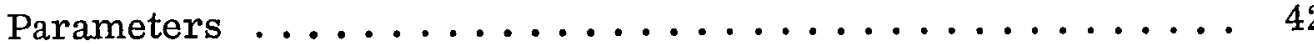


LIST OF FIGURES

Figure $\quad \underline{\text { Page }}$

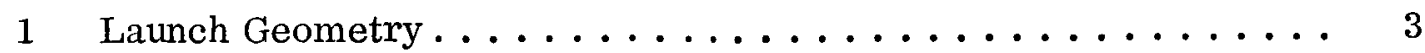

2 First Limit for Launch Trajectories . . . . . . . . . . . . . 5

3 Second Limit for Launch Trajectories . . . . . . . . . . . 6

4 Launch Velocity Diagram. .................... 10

5 Encounter Orbit Within the Sphere of Influence ........... 14

6 Planetary Approach Coordinate System and Impact Plane . . . . 16

7 Relationships in the Approach Coordinate System . . . . . . . 18

8 Vector Diagram of Cowell's Equations . . . . . . . . . . . . 21

9 Vector Diagram of Sun, Jupiter and Probe . . . . . . . . . 25

10 Constant Contours of Time to 10 A. U. . . . . . . . . . . . 43

A-1 Relationship Between True Anomaly and Eccentric Anomaly ... 48

B-1 Updating the Cartesian Coordinates . . . . . . . . . . . . . 50

$\mathrm{C}-1$ The Hyperbolic Orbit Geometry ................... 54 


\section{INTRODUCTION}

The ultimate goals of deep-space investigations are the understanding of the origin, laws and evolution of our solar system and of the galactic medium itself. By means of a probe to Jupiter and beyond into deep space we may extend our investigations many fold. Such a probe is currently being planned by NASA and will be entering a region never before investigated which will include the asteroid belt and the trapped radiation region at Jupiter.

A probe that is to be sent into deep space requires a tremendous amount of energy which with today's boosters can only be accomplished at the expense of the final payload. An imaginative way around this problem is to use the gravitational attraction of a planet. When a probe is in a free fall trajectory about the sun in such a manner that it passes within the vicinity of a planet, the trajectory is considerably changed by this encounter. The changes must, of course, depend on the encounter geometry of the probe and the planet.

This report considers a probe that will utilize a flyby of the planet Jupiter to obtain changes in the post encounter heliocentric trajectory. A by-product of an encounter with Jupiter is the opportunity to study the planet itself.

Many authors have treated a Jupiter flyby in the past (for example see references 8 thru 13) based on two body motion, however, Jupiter has twelve natural satellites, four of which are rather large and their masses are relatively well known. To the author's knowledge no one has done an integrated trajectory study which includes perturbations by the moons of Jupiter. In this report only the four Galilean moons are included since these are the largest and their masses are well known. The problem of obtaining accurate positions of these moons has resulted in the programming of Sampson's method to generate an ephemeris of the moons. Such a program has not previously been available in this country as far as can be determined. 


\section{THE TRANSFER TRAJECTORY}

The first problem that must be considered is that of the transfer trajectory from the earth to Jupiter. This will involve the determination of the proper launch conditions, parking orbit duration and injection energy to satisfy total flight time and nominal targeting at Jupiter.

Many authors have treated this problem using various methods to obtain a solution. References 1 through 7 in the bibliography give some of these methods and also indicate the complexity of the problem.

Since in the present study we are mainly interested in the probe once it is in the vicinity of Jupiter, the transfer orbit is only of minor interest. For this reason many of the constraints on the trajectory were dropped in order to simplify the solution. An approximate transfer trajectory is obtained using only the flight time and the positions of the launch and target bodies as constraints. No attempt is made to refine the approximate trajectory ir any way. The solution of the transfer trajectory under these restrictions is then solved using the well known solution to Lambert's problem. At the target planet the trajectory is altered by using the patched conic assumption which states that at any given time the probe is affected by one and only one body. At any time the gravitational field of a planet is considered to have a force field called the sphere of influence whose radius $s$ is given by (reference 1 )

$$
S=\left(\frac{m}{M}\right)^{2 / 5} R_{p}
$$

where $m$ and $M$ are the masses of the planet and sun respectively and $R_{p}$ is the planet's distance from the sun. 
During the transfer phase of the probe, the Earth and Jupiter are considered to be massless points until the probe comes within the sphere of influence of Jupiter. The method used to define this conic transfer trajectory is essentially that found in reference 7 and is described below.

Solution of the problem of a transfer trajectory necessitates knowing the heliocentric position vectors of the Earth $\left(\vec{r}_{e}\right)$ at launch date and of Jupiter $\left(\vec{r}_{j}\right)$ at arrival date. These vectors can be obtained from an ephemeris or by using two body equations and a given position of the planets at some epoch. Figure 1 shows the essential transfer geometry.

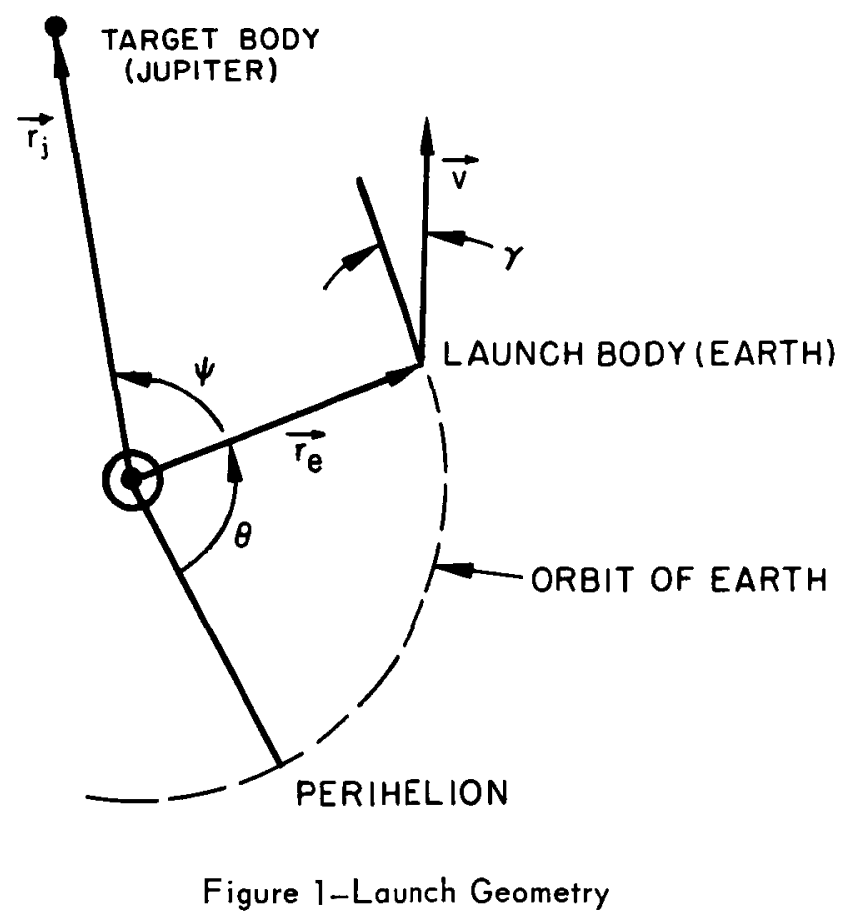

The heliocentric transfer angle $\Psi$ in figure 1 can be calculated from $\vec{r}_{e}$ and $\vec{r}_{j}$ and is the angle between the position vectors measured in the direction of planetary motion. Two other quantities in figure 1 to be defined are the angles $\theta$ and $\gamma$. Theta $(\theta)$ is the true anomaly of the Earth at launch and $\gamma$ is the 
heliocentric flight path angle which is the angle between a perpendicular to $\overrightarrow{\mathrm{r}}_{\mathrm{e}}$ and the velocity vector $\vec{v}$ of the probe at launch. The angle $\gamma$ is the parameter that we wish to calculate.

In order that the launch body and target body lie on a single conic orbit it is necessary that the following be true

$$
\begin{gathered}
\mathrm{r}_{\mathrm{e}}=\frac{\rho}{1+\mathrm{e} \cos \theta} \\
\mathrm{r}_{\mathrm{j}}=\frac{\rho}{1+\mathrm{e} \cos }(\theta+\Psi)
\end{gathered}
$$

where $\rho=\mathrm{a}\left(1-\mathrm{e}^{2}\right)$ is the semi latus rectum.

From (2)

$$
\rho=r_{j}[1+e \cos \theta \cos \Psi-e \sin \theta \sin \Psi]
$$

and from (1)

$$
\text { e } \cos \theta=\left(\frac{\rho}{r_{e}}-1\right)
$$

From figure (1)

$$
\begin{aligned}
\operatorname{Tan} \gamma=\cot (90-\gamma) & =\frac{\vec{r}_{e} \cdot \vec{v}}{\left|\vec{r}_{e} \times \vec{v}\right|} \\
& =\frac{\vec{r}_{e} \cdot \vec{v}}{\sqrt{a\left(1-e^{2}\right)}} \\
& =\frac{e \sin E}{\sqrt{1-e^{2}}}=\frac{r_{e} e \sin \theta}{\rho}
\end{aligned}
$$

Therefore

$$
\text { e } \sin \theta=\frac{\rho}{r_{e}} \operatorname{Tan} \gamma
$$

substituting (4) and (5) into (3) we obtain

$$
\rho=\frac{r_{e} r_{j}(1-\cos \Psi)}{r_{e}-r_{j} \cos \Psi+r_{j} \sin \Psi \operatorname{Tan} \gamma}
$$


Equation 6 characterizes the conic section connecting the two radius vectors and it is obvious that $\rho$ is a function only of the unknown angle $\gamma$.

The calculation of $\gamma$ is an interative procedure and only one value of $\gamma$ will yield the desired flight time between the two planets. Reference to figure 2 shows that two transfer trajectories of very short time exist. One is the straight line solution and the other bends very close around the sun (hot line). A solution lying inside the triangle formed by these two is forbidden since no conic section with the sun at a focus exists in this region. Thus a lower limit $\left(\gamma_{\mathrm{min}}\right)$ can be calculated for $\gamma$ by using the straight line solution and it is easily seen that

$$
\gamma_{\min }=\operatorname{Tan}^{-1}\left[\frac{r_{j} \cos \Psi-r_{e}}{r_{j} \sin \Psi}\right]
$$

The second limit for $\gamma$ is that for a posigrade parabolic orbit for an infinite transfer time. Reference to figure 3 shows the typical geometry for this orbit.

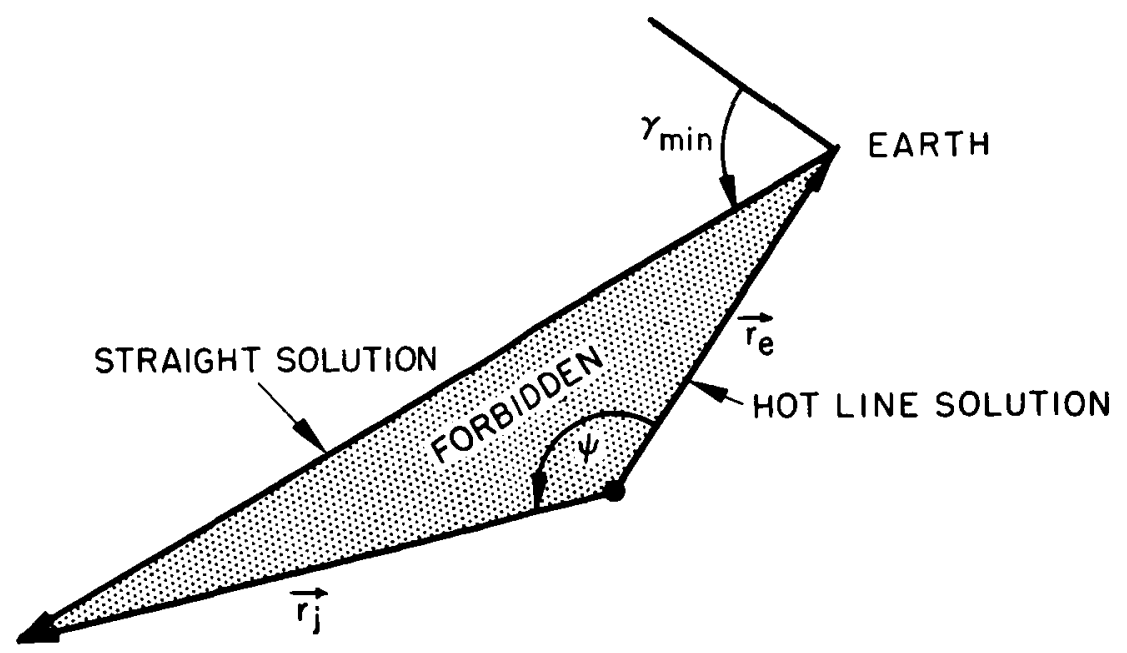

JUPITER

Figure 2-First Limit for Lounch Trajectories 


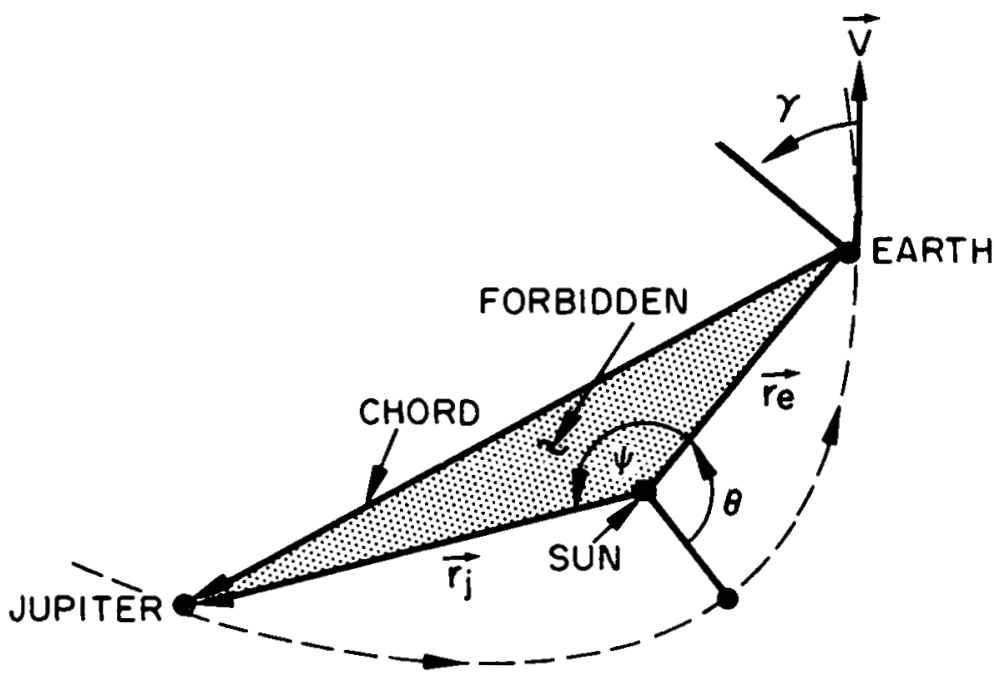

Figure 3-S econd Limit for Launch Trajectories

Recall equation (5)

$$
\mathrm{e} \sin \theta=\frac{\rho}{\mathrm{r}_{\mathrm{e}}} \operatorname{Tan} \gamma
$$

but for this orbit $\mathrm{e}=1$ and

$$
\rho=\frac{2 r}{1+\operatorname{Tan}^{2} \frac{\theta}{2}}
$$

Therefore

$$
\begin{aligned}
\sin \frac{\theta}{2} \cos \frac{\theta}{2} & =\frac{\operatorname{Tan} \gamma}{\frac{\cos ^{2} \frac{\theta}{2}+\sin ^{2} \frac{\theta}{2}}{\cos ^{2} \frac{\theta}{2}}} \\
& =\cos ^{2} \frac{\theta}{2} \operatorname{Tan} \gamma \\
\operatorname{Tan} \frac{\theta}{2} & =\operatorname{Tan} \gamma
\end{aligned}
$$

or 
Therefore for a parabola

$$
\theta=2 \gamma
$$

From equations (1) and (2) we have

$$
r_{e}(1+\cos \theta)=r_{j}[1+\cos (\theta+\Psi)]
$$

or

$$
r_{e}-r_{j}=\left(r_{j} \cos \Psi-r_{e}\right) \cos \theta-\left(r_{j} \sin \Psi\right) \sin \theta
$$

where $\theta$ is to be eliminated.

The square root of the sum of the squares of the coefficients of $\theta$ is:

$$
\left(r_{j}^{2}+r_{e}^{2}-2 r_{e} r_{j} \cos \Psi\right)^{1 / 2}=\text { chord }
$$

thus

$$
\frac{r_{e}-r_{j}}{\text { chord }}=\cos a \cos \theta-\sin a \sin \theta
$$

where

$$
\left.\begin{array}{l}
\cos \alpha=\frac{r_{j} \cos \Psi-r_{e}}{\operatorname{chord}} \\
\sin \alpha=\frac{r_{j} \sin \Psi}{\operatorname{chord}}
\end{array}\right\}
$$

Thus

$$
\frac{r_{e}-r_{j}}{\text { chord }}=\cos (\alpha+\theta)=\cos \beta
$$

and $a$ can be evaluated from eqn (9)

$$
\alpha=\operatorname{Tan}^{-1}\left[\frac{r_{j} \sin \Psi}{r_{j} \cos \Psi-r_{e}}\right]
$$


Therefore

$$
\alpha+\theta=\beta
$$

and from (8)

$$
\theta=2 \gamma
$$

Thus

$$
\gamma_{\text {LIM }}=\frac{\beta-\alpha}{2}
$$

Equations (7) and (10) now allow us to compute the transfer trajectory in the following manner:*

the initial guess for $\gamma$ is

$$
\gamma_{i}=\left\{\begin{array}{l}
\gamma_{\min } \text { if } \Psi<180^{\circ} \\
-\pi / 2 \text { if } \Psi>180^{\circ}
\end{array}\right.
$$

and a step size (ss) for $\gamma_{i}$ is chosen such that

$$
\gamma_{n}=\gamma_{n-1}+S S
$$

and

$$
\gamma_{\min }<\gamma_{\mathrm{n}}<\gamma_{1 \text { im }}
$$

From eqn (6)

$$
\rho=\frac{r_{e} r_{j}(1-\cos \Psi)}{r_{j} \sin \Psi \operatorname{Tan} \gamma_{n}-r_{j} \cos \Psi+r_{e}}
$$

The velocity is given by

$$
\begin{aligned}
\mathbf{v}^{2} & =\frac{2 \mu_{\mathrm{s}}}{\mathbf{r}_{\mathrm{e}}}-\frac{\mu_{\mathrm{s}}}{\mathrm{a}} \\
& =\frac{\rho \mu_{\mathrm{s}}}{\mathrm{r}_{\mathrm{e}}^{2} \cos ^{2} \gamma_{\mathrm{n}}}
\end{aligned}
$$

where $\mu_{\mathrm{s}}$ is the gravitational constant of the sun.

*These two limits on $y$ also rule out retrograde transfer orbits 
Define

$$
\mathrm{C} 3=\mathrm{v}^{2}-\frac{2 \mu_{\mathrm{s}}}{\mathrm{r}_{\mathrm{e}}}=-\frac{\mu_{\mathrm{s}}}{\alpha}
$$

which is twice the total energy per unit mass.

It can easily be shown that

$$
e \sin \theta=v \sin \gamma_{n} \sqrt{\frac{\rho}{\mu_{s}}}
$$

which yields $\theta$.

Then

$$
\begin{aligned}
& a=-\frac{\mu_{s}}{\mathrm{C} 3} \\
& e=\left(1-\frac{\rho}{a}\right)^{1 / 2}
\end{aligned}
$$

Appendix A shows how the time $\left(t_{e}\right)$ from periapsis to a true anomaly of $\theta$ can be calculated. In a similar manner the time $\left(t_{j}\right)$ from periapsis to Jupiter can be calculated. Then the flight time is

$$
\Delta \mathrm{t}=\mathrm{t}_{\mathrm{j}}-\mathrm{t}_{\mathrm{e}} \text {. }
$$

If $\Delta t$ is within a predetermined tolerance to the desired flight time then nothing more need be done. If not, the method of false position allows an iterative solution to $\gamma$ for the desired flight time. The quantities finally obtained are $\mathrm{v}, \mathrm{a}, \mathrm{e}, \theta$ and $\gamma$.

Using the above calculated $\gamma$ we may calculate the velocity vector $\vec{v}$ by decomposing onto two unit vectors $\vec{r}_{e}^{0}$ and $\left(\vec{r}_{e} \times \vec{r}_{j}\right)^{0} \times \vec{r}_{e}^{0}$ as in figure 4 . We obtain

$$
\overrightarrow{\mathbf{v}}=\frac{\mathbf{v}}{\mathbf{r}_{e}}\left[\cos \gamma\left(\overrightarrow{\mathbf{r}}_{e} \times \overrightarrow{\mathbf{r}}_{j}\right)^{0} \times \overrightarrow{\mathbf{r}}_{e}+\sin \gamma \overrightarrow{\mathbf{r}}_{e}\right]
$$




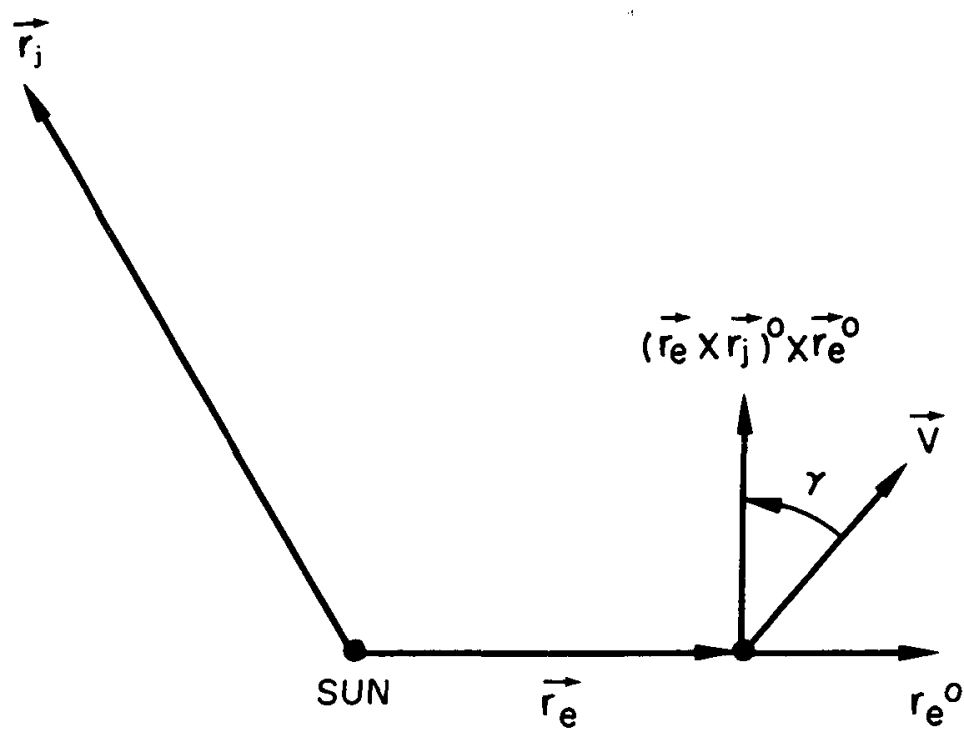

Figure 4-Launch Velocity Diagram

Equation 11 represents the velocity vector of the probe when it is launched from the Earth towards Jupiter. This velocity vector along with $\Psi$, the heliocentric transfer angle, and the position vector of the probe is all that is necessary to find the position and velocity of the probe at arrival at Jupiter. It is easily seen that for this approximate transfer trajectory, the position vector of the probe at launch is that of the Earth which is obtainable from the ephemeris. Appendix B describes the method used to update the position and velocity of the probe to the arrival date at Jupiter. 
As discussed in the last section the heliocentric velocity vector of the transfer orbit can be obtained at the arrival date at Jupiter. Since in this approximate scheme Jupiter is considered to be a massless point this arrival date is actually an impact time. The problem now is to determine the position and velocity of the probe at arrival at the sphere of influence of Jupiter. The heliocentric position vector of the probe at impact is obviously that of Jupiter.

Using the equation for the radius of the sphere of influence (S) discussed earlier

$$
S=\left(\frac{m}{M}\right)^{2 / 5} R_{p}
$$

the method of false position and two body equations allows us to back up the $\vec{r}$ and $\vec{v}$ from impact to a distance $S$ and thus obtain $\vec{r}$ and $\vec{v}$ at entrance to the sphere of influence.

We let $\vec{r}$ and $\vec{v}$ be the heliocentric impact position and velocity. From $\vec{r}$ and $\vec{v}$ we can easily compute the semi-major axis a, the eccentricity $e$, the mean motion $\mathrm{n}$ and the true anomaly $\theta_{i}$ at impact. From $\theta_{i}$ we find the eccentric anomaly $\mathrm{E}$ from

$$
\left.\begin{array}{l}
\operatorname{Sin} E=\frac{\sqrt{1-\mathrm{e}^{2}} \sin \theta_{i}}{1+e \cos \theta_{i}} \\
\operatorname{Cos} E=\frac{\cos \theta_{i}+e}{1+e \cos \theta_{i}}
\end{array}\right\}
$$


Kepler's equation then allows us to compute the time $t_{i}$ from periapsis to $\theta_{i}$

$$
M=E-e \sin E=n t_{i}
$$

The next step is to start the false position interation by choosing a time

$$
t=t_{i}-\Delta t_{n}
$$

where $\Delta t_{n}$ can be any arbitrary time.

Kepler's equation is then solved for $\mathrm{E}$ in an interative procedure as

$$
E_{n}=M+e \sin E_{n-1}
$$

where $E_{o}=M$ and $M=n t$

The true anomaly $\theta$ at time $\mathrm{t}$ is then found from

$$
\left.\begin{array}{l}
\sin \theta=\frac{\sqrt{1-e^{2}} \sin E}{1-e \cos E} \\
\cos \theta=\frac{\cos E-e}{1-e \cos E}
\end{array}\right\}
$$

We now have $\delta=\theta_{i}-\theta$ and using the procedure in Appendix B we may find $\vec{r}_{n}, \vec{v}_{n}$ the position and velocity at time $t$.

Define a function $F$

$$
\mathrm{F}=\mathrm{D}-\mathrm{S}
$$

where $D$ is the distance from Jupiter which is obtained from $\vec{r}_{n}$ and the ephemeris of Jupiter. A new $\Delta t_{n+1}$ is then calculated from

$$
\Delta t_{n+1}=\frac{\Delta t_{n-1} F_{n}-\Delta t_{n} F_{n-1}}{F_{n}-F_{n-1}}
$$

where of course at the beginning

$$
\Delta \mathbf{t}_{0}=0
$$

and

$$
F_{n-1}=-S \text {. }
$$


We then return to equation (14) and repeat the procedure until the function $F$ is within a predetermined small value.

The Undisturbed Post Encounter Trajectory

We have now determined the heliocentric position and velocity $\vec{r}$ and $\vec{v}$ of the probe when it enters Jupiter's sphere of influence. The next problem is to find the post encounter orbit of the probe after it has passed thru the sphere of influence as a function of encounter geometry. References 8 thru 13 describes various methods used to obtain the post encounter orbit as a function of encounter conditions. The method described below is that used by the authors of references 8 and 9 with some modification to make the method more accurate.

We have $\vec{v}$ from above and knowing the injection date of the probe and the time to the sphere of influence we can find $\vec{v}_{p}$ the heliocentric velocity of Jupiter from the ephemeris data. The velocity of the probe with respect to Jupiter at entrance to the sphere of influence, $\vec{v}_{1}^{\prime}$, is then given by

$$
\vec{v}_{1}^{\prime}=\vec{v}-\vec{v}_{p}
$$

as can be seen from a diagram of the velocity vectors.

Within the sphere of influence the probe's trajectory will be hyperbolic with respect to the planet (reference 1 - see figure 5). We assume that we may choose the radius of closest approach $\mathbf{r}_{\mathbf{p}}$ of the probe without significantly altering the direction or magnitude of $\vec{v}_{1}^{\prime}$. This is equivalent to assuming that while the direction and magnitude of $\vec{v}_{1}^{\prime}$ are fixed at the calculated values, we are still free to choose the point at which the probe pierces the surface of the sphere of influence (point $A$ in figure 5). That this assumption is valid is indicated by Table 1 (from reference 8 ) where $\vec{v}_{1}^{\prime}$ lies along the $\vec{S}^{\circ}$ vector (figure 5). This table was compiled for both retrograde and posigrade flybys of Jupiter and for a radius of closest approach $r_{p}$ varying from 100,000 to $10,000,000 \mathrm{~km}$. These values for $\mathbf{r}_{\mathbf{p}}$ span a far larger region than we are interested in. 


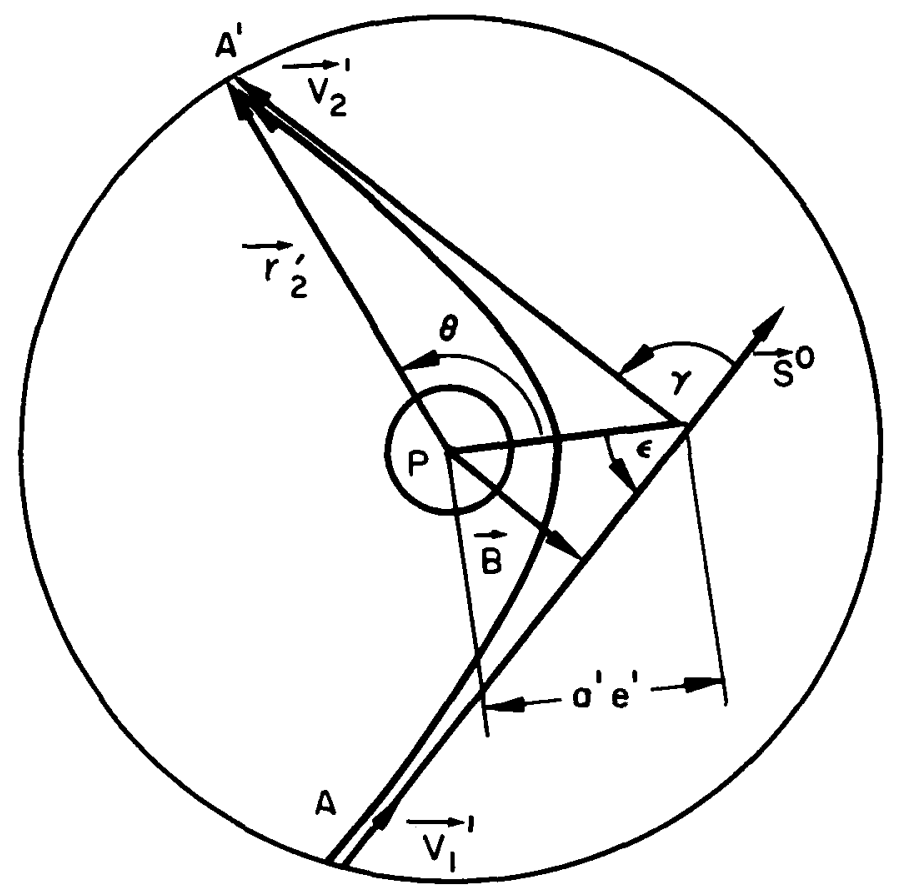

Figure 5-Encounter Orbit Within the Sphere of Influence (From Reference 8)

For a given asymptote and energy at entrance it will be shown that the entire trajectory within the sphere of influence, including $r_{p}$, is determined by the location of point $A$.

A convenient method for specifying the location of point A (reference 8, 9 and 11) and the radius of closest approach is to select the magnitude of the miss vector $(|\vec{B}|=$ B in figure 5) and an angle $\Psi$, measured between $\vec{B}$ and a fixed vector in the planet's orbital plane. This fixed vector $\overrightarrow{\mathrm{T}}^{\circ}$ is one axis of an orthogonal coordinate system whose origin is the planet's center and consists of the unit vectors $\overrightarrow{\mathrm{S}}^{\circ}, \overrightarrow{\mathrm{T}}^{\circ}$ and $\overrightarrow{\mathrm{R}}^{\circ}$ (References 10,11, 14 and 15). These vectors can be defined in the following manner:

$$
\vec{S}^{\circ}=\frac{\vec{v}_{1}^{\prime}}{\left|\vec{v}_{1}^{\prime}\right|}
$$


Table I

The Velocity Vector as a Function of Choosing the Entrance Point on the Sphere of Influence (from reference 8)

\begin{tabular}{|c|c|c|c|}
\hline \multirow{2}{*}{$\begin{array}{c}\text { Posigrade } \\
\mathrm{r}_{\mathrm{p}} \times 10^{5} \mathrm{~km}\end{array}$} & $\begin{array}{c}|c| \\
\mathrm{S}^{\circ}\end{array}$ & $\begin{array}{c}\left|\overrightarrow{\mathrm{V}}_{1}\right| \\
(\mathrm{km} / \mathrm{sec})\end{array}$ \\
\cline { 2 - 4 } & Right Ascension & $\begin{array}{c}\text { Declination } \\
(\mathrm{DEG})\end{array}$ & \\
\hline 1 & 161.38295 & -.037895218 & 13.863505 \\
5 & 161.36287 & -.037826118 & 13.873625 \\
10 & 161.34038 & -.037764311 & 13.882193 \\
50 & 161.10998 & -.037458739 & 13.910651 \\
100 & 160.75975 & -.037179910 & 13.922712 \\
\hline Retrograde & & & \\
1 & 161.43148 & -.037922481 & 13.863560 \\
5 & 161.48549 & -.037896001 & 13.873828 \\
10 & 161.53529 & -.037877017 & 13.882264 \\
50 & 161.82623 & -.037871506 & 13.910774 \\
100 & 162.11483 & -.037958746 & 13.923062 \\
\hline
\end{tabular}

$\overrightarrow{\mathrm{S}}^{\circ}$ is assumed to lie along the incoming asymptote of the hyperbola;

$$
\overrightarrow{\mathrm{T}}^{\circ}=\overrightarrow{\mathrm{k}}^{\circ} \times \overrightarrow{\mathrm{S}}^{\circ}
$$

where $\overrightarrow{\mathrm{k}}^{\circ}$ is a unit vector perpendicular to the planet's orbital plane;

$$
\overrightarrow{\mathbf{R}}^{\circ}=\overrightarrow{\mathbf{S}}^{\circ} \times \overrightarrow{\mathbf{T}}^{\circ}
$$

The vector $\overrightarrow{\mathrm{T}}^{\circ}$ lies in the planet's orbital plane and is positive in the direction of the planet's motion. We define $\Psi$ to be the angle between $\vec{T}^{\circ}$ and $\vec{B}$ measured in the direction $\overrightarrow{\mathrm{T}}^{\circ}$ to $\overrightarrow{\mathrm{R}}^{\circ}$. Figure 6 (From ref. 14) shows the geometry of this coordinate system and its relationship to the impact plane which is perpendicular to the incoming asymptote. 


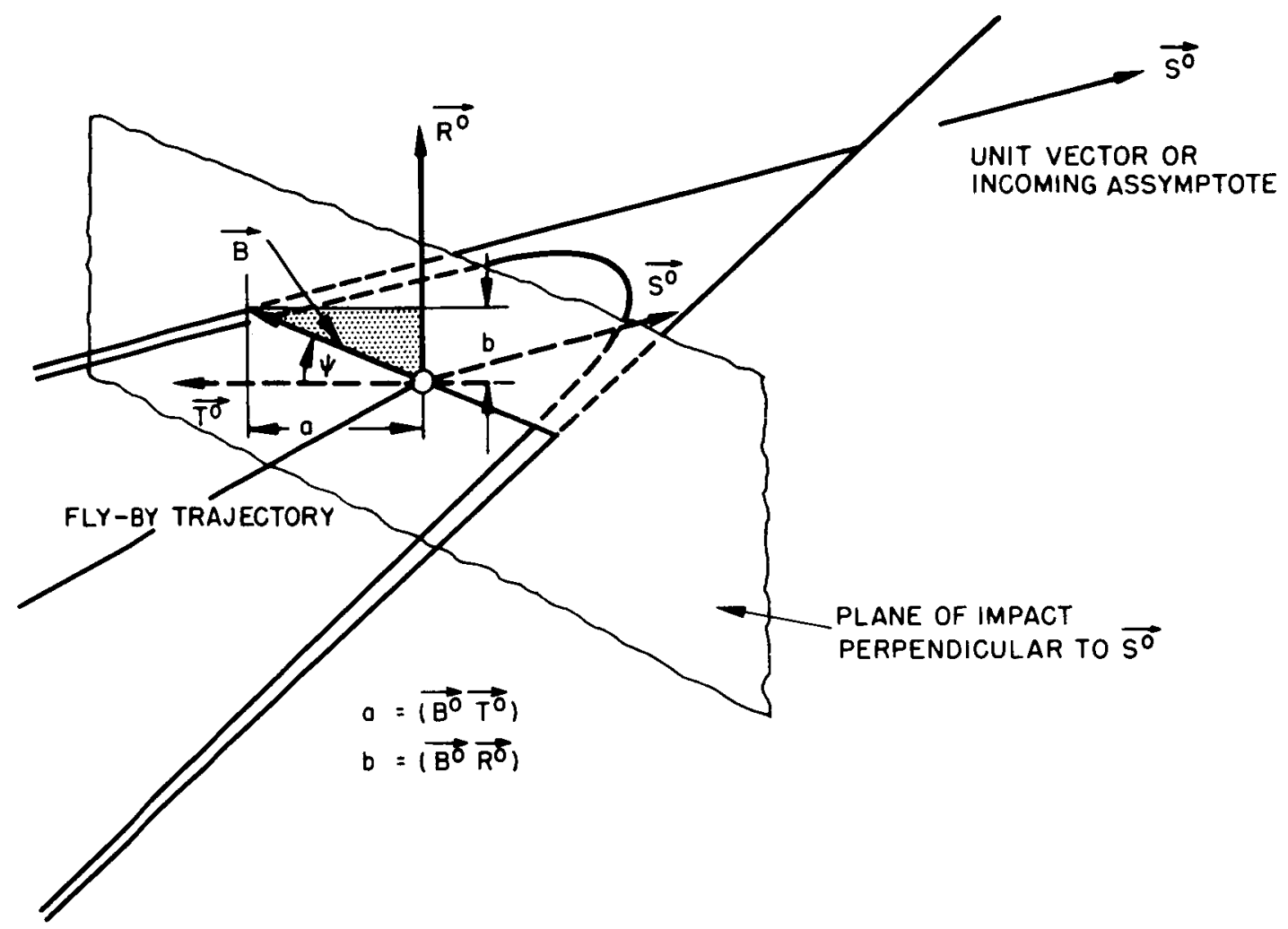

Figure 6-Planetary Approach Coordinate System and Impact Plane (From Reference 14)

The total effect of the undisturbed encounter is simply to rotate $\vec{v}_{1}^{\prime}$, thru an angle $\gamma$, as illustrated in figure 5 , since we must have

$$
\left|\vec{v}_{1}^{\prime}\right|=\left|\vec{v}_{2}^{\prime}\right|
$$

if energy is to be conserved (references 1,8 and 11). We may easily calculate $\gamma$ if $B$ is specified:

let a be the semi major axis of the hyperbolic orbit. Then from a straight forward rearrangement of the energy equation we have

$$
\mathrm{a}=\frac{\mu_{\mathrm{p}} \mathrm{S}}{2 \mu_{\mathrm{p}}-\mathrm{S}\left|\overrightarrow{\mathbf{v}}_{1}^{\prime}\right|^{2}}
$$


where $\mu_{\mathrm{p}}$ is the planet's gravitational constant. From figure 5 and the properties of an hyperbola we have the eccentricity

$$
\mathrm{e}=\frac{1}{\cos \epsilon}
$$

where $\epsilon$ is the half angle between the asymptotes and

$$
\sin \epsilon=\frac{|\vec{B}|}{a \mathrm{e}}=\frac{\mathrm{B}}{\mathrm{a}} \cos \epsilon \text {. }
$$

Therefore

$$
\operatorname{Tan} \epsilon=\frac{\mathrm{B}}{\mathrm{a}}
$$

now

$$
\begin{aligned}
& \gamma=\pi-2 \epsilon \quad \text { or } \\
& \gamma=\pi-2 \operatorname{Tan}^{-1}\left(\frac{B}{a}\right)
\end{aligned}
$$

and the radius of closest approach is

$$
\mathbf{r}_{p}=a(1-e)
$$

We have already pointed out how we may choose the point of entry on the sphere of influence without altering the direction or magnitude of $\vec{v}_{1}^{\prime}$. However, this obviously will change $\vec{r}_{1}^{\prime}$ the Jovian centered position vector of the probe. We have shown how the parameters $B$ and $\Psi$ define the entrance point on the sphere of influence and allow the calculation of the hyperbolic orbit. We can use $B$ and $\Psi$ to obtain $\vec{r}_{1}^{\prime}$ from which we will then be able to easily calculate the post encounter orbit at exit from the sphere of influence. We will also use $\vec{v}_{1}^{\prime}$ and the resulting $\vec{r}_{1}^{\prime}$ as starting conditions for the calculation of the disturbed orbit in the next section.

Figure 7 shows the relationship of the vectors and angles involved in the $\vec{R}^{\circ}, \vec{S}^{\circ}, \vec{T}^{\circ}$ coordinate system. 


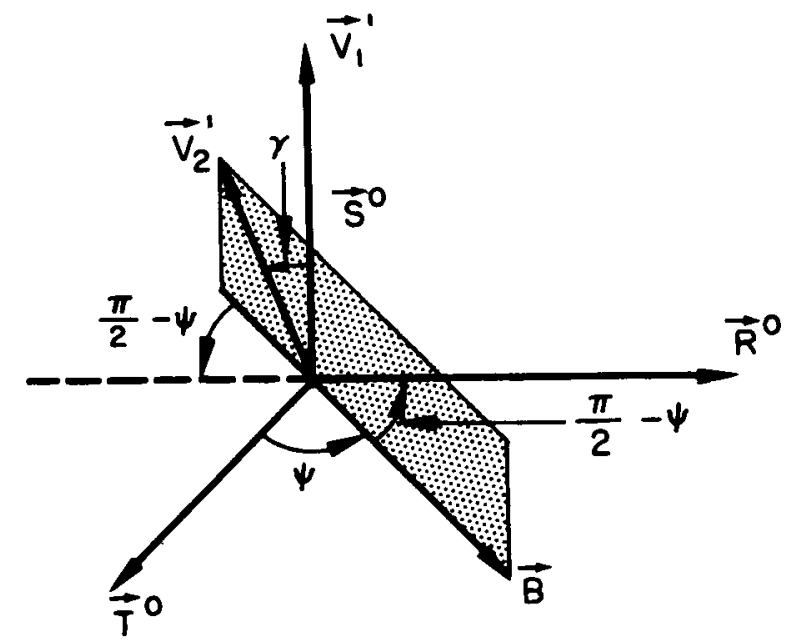

Figure 7-Relationships in the Approach Coordinate System (From Reference 8)

Referring to this figure we see that

$$
\vec{B}^{\circ}=\vec{T}^{\circ} \cos \Psi+\vec{R}^{\circ} \sin \Psi
$$

From equations (19) and (20) we find the semi latus rectum

$$
\rho=\left|a\left(e^{2}-1\right)\right|
$$

and the true anomaly at entrance to the sphere of influence

$$
\theta=\cos ^{-1} \frac{\rho-\mathrm{S}}{\mathrm{Se}}
$$

Now from figure 5 we find $\vec{r}_{1}^{\prime}$, the Jovian centered position vector at entrance to the sphere of influence

$$
\overrightarrow{\mathbf{r}}_{1}^{\prime}=\mathrm{S}\left[\cos (\theta+\epsilon-\pi / 2) \overrightarrow{\mathrm{B}}^{\circ}+\cos (\theta+\epsilon) \overrightarrow{\mathrm{S}}^{\circ}\right]
$$

Equation (26) however does not represent the true position vector since it was assumed that $\vec{v}_{1}^{\prime}$ represents the incoming asymptote

$$
\overrightarrow{\mathrm{S}}^{\circ}=\frac{\overrightarrow{\mathrm{v}}_{1}^{\prime}}{\left|\overrightarrow{\mathrm{v}}_{1}^{\prime}\right|}
$$


$\overrightarrow{\mathrm{S}}^{\circ}$ is therefore not the true asymptote and the miss vector $\overrightarrow{\mathrm{B}}$ is not quite perpendicular to $\overrightarrow{\mathrm{S}}^{\circ}$. The position vector $\overrightarrow{\mathrm{r}}_{1}^{\prime}$ is thus not quite compatible with the anomaly $\theta$. We may make the entire orbit compatible by using $\vec{r}_{1}^{\prime}$ to calculate a new $\theta$ and a new time in the sphere of influence as follows: the semi latus rectum is

$$
\rho=\frac{\left|\vec{r}_{1}^{\prime} \times \overrightarrow{\mathbf{v}}_{\mathbf{i}}\right|^{2}}{\mu_{\mathrm{p}}}
$$

then

$$
\begin{aligned}
\mathrm{C} 3 & =\left|\overrightarrow{\mathrm{v}}_{1}^{\prime}\right|^{2}-\frac{2 \mu_{\mathrm{p}}}{\mathrm{r}_{1}^{\prime}} \\
\mathrm{a} & =-\frac{\mu_{\mathrm{p}}}{\mathrm{C} 3} \\
\mathrm{e} & =\sqrt{1+\frac{\mathrm{C} 3 \rho}{\mu_{\mathrm{p}}}}
\end{aligned}
$$

and finally

$$
\theta=\cos ^{-1}\left(\frac{\rho-\mathrm{r}_{1}^{\prime}}{\mathrm{r}_{1}^{\prime} \mathrm{e}}\right) \text {. }
$$

The procedure in Appendix A then yields the time from perijove to the sphere of influence which is one half the total time spent in the sphere. The differences in total time spent in the sphere of influence between this new trajectory and the approximate trajectory is on the order of 10 minutes when the total time is approximately 80 days and the magnitude $B$ of the miss vector changes by less than one percent. Since B was one of the parameters which define the encounter orbit and therefore is chosen beforehand, any change in B will slightly alter the encounter trajectory. But since the change in B is small any changes produced by making the orbit consistent with $\vec{r}_{1}^{\prime}$ are negligible. Table 2 shows the differences, resulting from this procedure, obtained in the magnitude of the miss vector and the total time in the sphere of influence. 
Table II

Effects of Approximating the Encounter Trajectory

\begin{tabular}{|r|c|c|l|c|c|c|}
\hline \multicolumn{1}{|c|}{$\psi$} & Old B $(\mathrm{km})$ & New B $(\mathrm{km})$ & $\Delta(\mathrm{km})$ & Old Time (days) & New Time (days) & $\Delta$ (days) \\
\hline \hline $180^{\circ}$ & $1.25 \times 10^{6}$ & $1.26 \times 10^{6}$ & $.01 \times 10^{6}$ & 83.009384 & 83.017077 & .0077 \\
$0^{\circ}$ & $1.25 \times 10^{6}$ & $1.26 \times 10^{6}$ & $.01 \times 10^{6}$ & 83.009384 & 83.017128 & .0077 \\
$180^{\circ}$ & $1.0 \times 10^{6}$ & $1.0078 \times 10^{6}$ & $.0078 \times 10^{6}$ & 81.453790 & 81.460214 & .0064 \\
$0^{\circ}$ & $1.0 \times 10^{6}$ & $1.0078 \times 10^{6}$ & $.0078 \times 10^{6}$ & 81.453790 & 81.460217 & .0064 \\
$180^{\circ}$ & $5.0 \times 10^{6}$ & $5.0389 \times 10^{6}$ & $.0389 \times 10^{6}$ & 82.912777 & 82.915892 & .0031 \\
$0^{\circ}$ & $5.0 \times 10^{6}$ & $5.0388 \times 10^{6}$ & $.0388 \times 10^{6}$ & 82.912777 & 82.915926 & .0031 \\
\hline
\end{tabular}

With the now determined Jovian centered position and velocity $\vec{r}_{1}^{\prime}$ and $\vec{v}_{1}^{\prime}$ and the total time $t$ spent in the sphere of influence we are in a position to find $\vec{r}_{2}^{\prime}$ and $\vec{v}_{2}^{\prime}$ the Jovian centered position and velocity of the probe at exit from the sphere (Point $A^{\prime}$ in figure 5). To do this one needs an accurate means of stepping along the hyperbola. The method used to determine $\vec{r}_{2}^{\prime}$ and $\vec{v}_{2}^{\prime}$ is described fully in Appendix C.

Using $\mathrm{t}$ the time in the sphere of influence we can determine the position and velocity of Jupiter from ephemeris information at the time the probe leaves the sphere of influence. This position and velocity with $\vec{r}_{2}^{\prime}$ and $\vec{v}_{2}^{\prime}$ determines the heliocentric position and velocity of the post encounter trajectory. 


\section{THE PERTURBED JUPITER FLYBY}

As pointed out in the previous section the Jovian centered $\vec{r}_{1}^{\prime}$ and $\vec{v}_{1}^{\prime}$ obtained there are also to be used as starting conditions for the disturbed trajectory. The disturbed trajectory is treated in the standard manner using either Cowell's or Encke's method. A review of these methods is given below.

\section{Cowell's Equations of Motion - Perturbations in Rectangular Coordinates}

Figure 8 shows three bodies, S, P and $P^{\prime}$ with masses $M, m$ and $m^{\prime}$ respectively. The point 0 is a fixed point in space. We consider $P$ to be the body which is in motion about $\mathrm{S}$ and is disturbed by body $\mathrm{P}^{\prime}$.

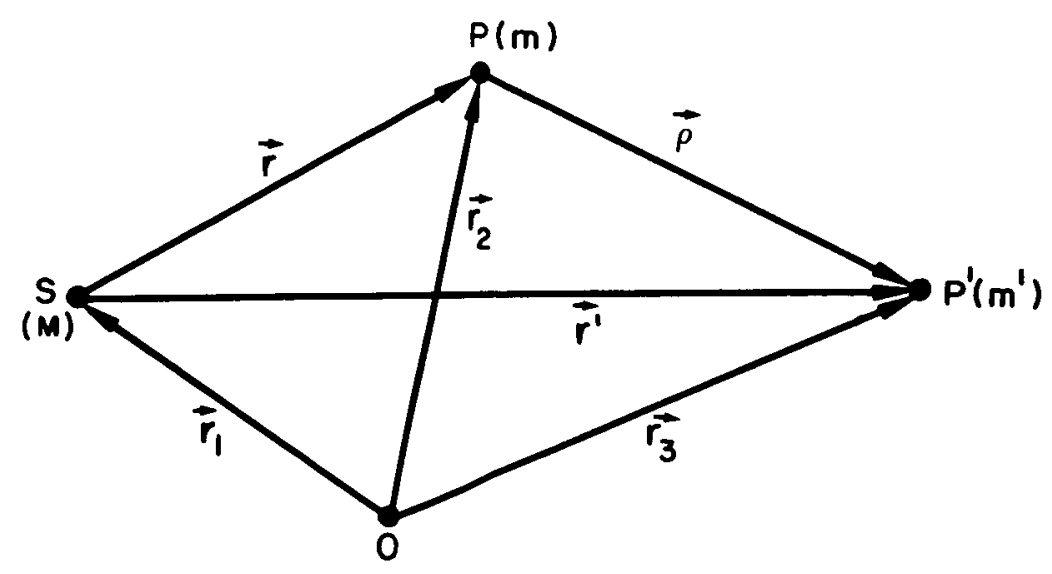

Figure 8-Vector Diagram of Cowell's Equations 
Referring everything to point 0 we may express the force on $\mathbf{S}$ as

$$
M \frac{d^{2} \vec{r}_{1}}{d t^{2}}=\frac{k^{2} M m}{\left|\vec{r}_{1}-\vec{r}_{2}\right|^{3}}\left(\vec{r}_{2}-\vec{r}_{1}\right)+\frac{k^{2} M^{\prime}}{\left|\vec{r}_{1}-\vec{r}_{3}\right|^{3}}\left(\vec{r}_{3}-\vec{r}_{1}\right)
$$

The force on $\mathrm{P}$ is

$$
\frac{m d^{2} \vec{r}_{2}}{d t^{2}}=\frac{k^{2} M m}{\left|\vec{r}_{2}-\vec{r}_{1}\right|^{3}}\left(\vec{r}_{1}-\vec{r}_{2}\right)+\frac{k^{2} m m^{\prime}}{\left|\vec{r}_{3}-\vec{r}_{2}\right|^{3}}\left(\vec{r}_{3}-\vec{r}_{2}\right)
$$

we define

$$
\begin{aligned}
\vec{r} & =\vec{r}_{2}-\vec{r}_{1} \\
\vec{r}^{\prime} & =\vec{r}_{3}-\vec{r}_{1} \\
\vec{r}^{\prime}-\vec{r} & =\vec{r}_{3}-\vec{r}_{2}=\vec{\rho}
\end{aligned}
$$

By subtracting equation (27) from (28) and making the above substitutions we obtain

$$
\frac{d^{2} \vec{r}}{d t^{2}}=-\frac{k^{2}(M+m)}{r^{3}} \vec{r}+k^{2} m^{\prime}\left(\frac{\vec{r}^{\prime}-\vec{r}}{\rho^{3}}-\frac{\vec{r}^{\prime}}{r^{\prime 3}}\right)
$$

This is Cowell's equation of motion in rectangular coordinates and is now referenced to the central body $S$. If there is more than one disturbing body the above equation may be written as:

$$
\frac{d^{2} \vec{r}}{d^{2}}=-\frac{k^{2}(M+m)}{r^{3}} \vec{r}+\sum_{i} k^{2} m_{i}^{\prime}\left(\frac{\vec{r}_{i}^{\prime}-\vec{r}}{\rho_{i}^{3}}-\frac{\vec{r}_{i}^{\prime}}{r_{i}^{\prime 3}}\right)
$$

\section{Encke's Equations of Motion}

In the above development if the acceleration due to the disturbing bodies is of the same or higher order as that due to the central force field then Cowell's procedure is a good one to use (reference 1). If, however, the disturbing acceleration is small then Cowell's method becomes inefficient. If in this case 
only the differential accelerations are integrated then efficiency and accuracy may be retained. This procedure is the well-known Encke's method.

Define

$$
\vec{r}=\vec{r}_{0}+\delta \vec{r}
$$

where $\overrightarrow{\mathbf{r}}_{0}$ is the two body position vector and $\delta \vec{r}$ is the differential correction. The equation of motion is then

$$
\frac{d^{2} \vec{r}}{d t^{2}}=-\frac{k^{2} M \vec{r}}{r^{3}}+\vec{F}
$$

where $\vec{F}$ is the disturbing acceleration.

For the two body case we have

$$
\frac{\mathrm{d}^{2} \overrightarrow{\mathrm{r}}_{0}}{\mathrm{dt^{2 }}}=-\frac{\mathrm{k}^{2} \mathrm{M} \overrightarrow{\mathrm{r}}_{0}}{\mathrm{r}_{0}{ }^{3}}
$$

Upon subtracting (32) from (31) we obtain

$$
\frac{d^{2} \delta \vec{r}}{d t^{2}}=-\frac{k^{2} M \delta \vec{r}}{r_{0}^{3}}+k^{2} M\left(\frac{1}{r_{0}^{3}}-\frac{1}{r^{3}}\right) \vec{r}+\vec{F}
$$

Denote

$$
\frac{\mathrm{k}^{2} \mathrm{M}}{\mathrm{r}_{0}^{3}}=\mathrm{h}
$$

and

$$
\frac{r^{2}}{r_{0}^{2}}=1+2 q
$$

Observe that since $r^{2}$ and $r_{0}^{2}$ are nearly equal, $2 q$ will be small.

Now

$$
\begin{aligned}
1+2 q & =\frac{\overrightarrow{\mathbf{r}} \cdot \overrightarrow{\mathbf{r}}}{\mathbf{r}_{0}^{2}}=\frac{\left(\overrightarrow{\mathbf{r}}_{0}+\delta \overrightarrow{\mathbf{r}}\right) \cdot\left(\overrightarrow{\mathbf{r}}_{0}+\delta \overrightarrow{\mathbf{r}}\right)}{\mathbf{r}_{0}^{2}} \\
& =\frac{\mathbf{r}_{0}^{2}+2 \overrightarrow{\mathbf{r}}_{0} \cdot \delta \overrightarrow{\mathbf{r}}+\delta \overrightarrow{\mathbf{r}} \cdot \delta \overrightarrow{\mathbf{r}}}{\mathbf{r}_{0}^{2}} .
\end{aligned}
$$


Therefore

$$
q=\frac{\left(\vec{r}_{0}+1 / 2 \delta \vec{r}\right) \cdot \delta \vec{r}}{r_{0}^{2}}
$$

and

$$
\frac{r_{0}^{3}}{r^{3}}=(1+2 q)^{-3 / 2}
$$

Then equation (33) becomes upon substituting (34) and (36)

$$
\frac{\mathrm{d}^{2} \delta \overrightarrow{\mathbf{r}}}{d \mathrm{t}^{2}}=-\mathrm{h} \delta \overrightarrow{\mathbf{r}}+\mathrm{h}\left[1-(1+2 \mathrm{q})^{-3 / 2}\right] \overrightarrow{\mathbf{r}}+\overrightarrow{\mathrm{F}}
$$

Observe that

$$
1-(1+2 q)^{-3 / 2}=q\left(3-\frac{15}{2} q+\cdots\right)=f q
$$

then

$$
\frac{d^{2} \delta \vec{r}}{d t^{2}}=-h \delta \vec{r}+h f q \vec{r}+\vec{F}
$$

Equation (38) is the form of Encke's equations used for computation. However, Encke's equations do have a disadvantage that causes computation time to increase. When the magnitude of $\delta \vec{r}$ in equation (38) becomes large it is necessary to change the two body vector $\vec{r}_{0}$ by defining $\vec{r}_{0}=\vec{r}$ if accuracy is to be maintained. This process, known as rectification, requires the numerical integrator to be restarted with a resulting increase in time. It should be pointed out that the disturbing acceleration $\vec{F}$ is the same as in equation (30) above; i.e.,

$$
\vec{F}=\sum_{i} k^{2} m_{i}^{\prime}\left(\frac{\vec{r}_{i}^{\prime}-\vec{r}}{\rho_{i}^{3}}-\frac{\vec{r}_{i}^{\prime}}{r_{i}^{\prime 3}}\right)
$$

and that the only change has been in the central body term of equation (30). 
Perturbations Due to the Sun

In the above equation (39) for the disturbing acceleration we will include only the four great moons of Jupiter. Since the distance of the sun from Jupiter is so much larger than that of the moons, the term in parentheses in equation (39) for the sun will lose accuracy because of the fact that both members are almost equal. We may eliminate this problem in a manner similar to Encke's procedure by treating the sun as a special case. Reference 16 describes a method for solving this problem and the procedure is repeated below.

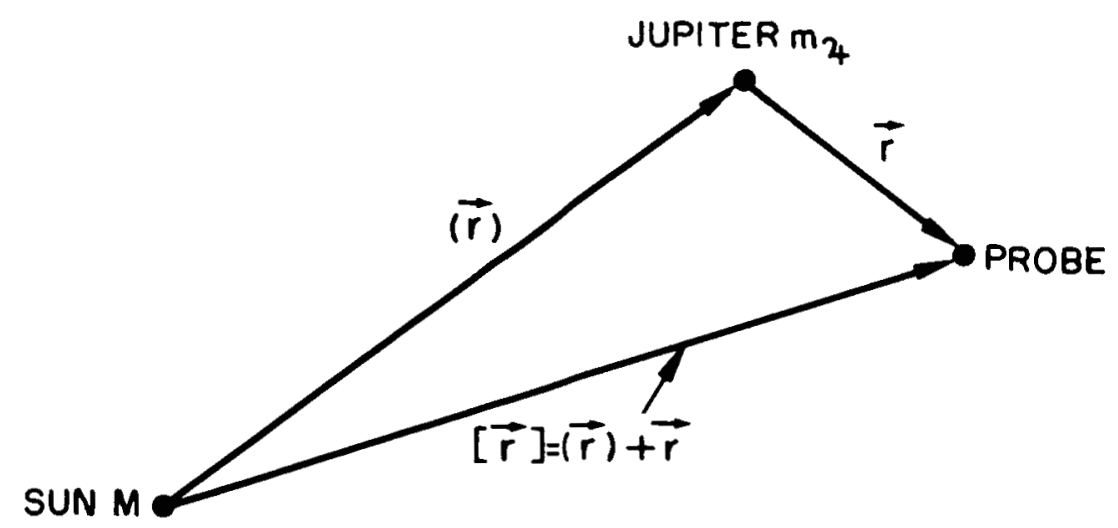

Figure 9-Vector Diagram of Sun, Jupiter and Probe

Figure 9 shows the geometry of the sun, Jupiter and Probe during the encounter phase. By a slight change in notation we may write equation (29) for the case where Jupiter is the central body and the sun is the disturbing body:

$$
\frac{d^{2} \vec{r}}{d t^{2}}=-\frac{k^{2} m_{4}}{r^{3}} \vec{r}+k^{2} M\left(\frac{-(\vec{r})-\vec{r}}{|(\vec{r})+\vec{r}|^{3}}-\frac{-(\vec{r})}{|(\vec{r})|^{3}}\right)
$$

or

$$
\frac{d^{2} \vec{r}}{d t^{2}}=-\frac{k^{2} m_{4}}{r^{3}} \vec{r}-k^{2} M\left(\frac{(\vec{r})+\vec{r}}{|(\vec{r})+\vec{r}|^{3}}-\frac{(\vec{r})}{|(\vec{r})|^{3}}\right)
$$

where $\vec{r}$ and $(\vec{r})$ are defined as in figure 9 and the notational change is obvious by comparing figures 8 and 9 . 
Now

$$
|(\vec{r})+\vec{r}|^{2}=|(\vec{r})|^{2}+2(\vec{r}) \cdot \vec{r}+|\vec{r}|^{2}
$$

let

$$
q=\frac{\frac{1}{2} r^{2}+(\vec{r}) \cdot \vec{r}}{|(\vec{r})|^{2}}
$$

then

$$
2|(\vec{r})|^{2} q=r^{2}+2(\vec{r}) \cdot \vec{r}
$$

upon substituting this into equation (41) we obtain

$$
\begin{aligned}
|(\vec{r})+\vec{r}|^{2} & =|(\vec{r})|^{2}+2|(\vec{r})|^{2} q \\
& =|(\vec{r})|^{2}(1+2 q)
\end{aligned}
$$

then

$$
|(\vec{r})+\vec{r}|^{-3}=\frac{1}{|(\vec{r})|^{3}}(1+2 q)^{-3 / 2}
$$

let

$$
\left.\begin{array}{c}
h=\frac{k^{2} m_{4}}{r^{3}} \\
(h)=\frac{k^{2} M}{|(\vec{r})|^{3}}
\end{array}\right\}
$$

Substituting equations (43) and (44) into (40) yields

$$
\begin{aligned}
\frac{d^{2} \vec{r}}{{d t^{2}}^{2}} & =-h \vec{r}-k^{2} M\left\{((\vec{r})+\vec{r}) \frac{1}{|(\vec{r})|^{3}}(1+2 q)^{-3 / 2}-\frac{(\vec{r})}{|(\vec{r})|^{3}}\right\} \\
& =-h \vec{r}-(h)[(\vec{r})+\vec{r}](1+2 q)^{-3 / 2}+(h)(\vec{r}) \\
\frac{d^{2} \vec{r}}{d^{2}} & =\left[-h-(h)(1+2 q)^{-3 / 2}\right] \vec{r}+(h)(\vec{r})\left[-(1+2 q)^{-3 / 2}+1\right]
\end{aligned}
$$


now making the same series expansion as before

$$
\begin{aligned}
1-(1+2 \mathrm{q})^{-3 / 2} & =\mathrm{q}\left(3-\frac{15}{2} \mathrm{q}+\frac{35}{2} \mathrm{q}^{2}+\cdots\right) \\
& =\mathrm{fq}
\end{aligned}
$$

the equations of motion become

$$
\frac{\mathrm{d}^{2} \overrightarrow{\mathbf{r}}}{\mathrm{dt^{2 }}}=\{-\mathrm{h}-(\mathrm{h})[1-\mathrm{fq}]\} \overrightarrow{\mathbf{r}}+(\mathrm{h})(\overrightarrow{\mathbf{r}}) \mathrm{fq}
$$

or

$$
\frac{d^{2} \vec{r}}{d t^{2}}=\{(h) f q-(h)-h\} \vec{r}+(h) f q(\vec{r})
$$

where the term due solely to the central body is $h \vec{r}$ and the rest is due to the presence of the sun.

Combining the Equations of Motion

The equations of motion are now combined to include the perturbations due to the sun and those due to other bodies. Rewrite equation (46) in the following form

$$
\frac{d^{2} \vec{r}}{d t^{2}}=-h \vec{r}+\phi
$$

where $\phi$ is the acceleration due to the presence of the sun

$$
\phi=\{(h) f q-(h)\} \vec{r}+(h) f q(\vec{r})
$$

and

$$
\mathrm{h}=\frac{\mathrm{k}^{2} \mathrm{~m}_{4}}{\mathrm{r}^{3}}
$$

where $r$ is the distance from Jupiter to the probe. 
To include other bodies we must add to equation (47) the acceleration expressed as $\vec{F}$ in equation (39)

$$
\frac{d^{2} \vec{r}}{d t^{2}}=-h \vec{r}+\phi+\vec{F}
$$

where

$$
\overrightarrow{\mathbf{F}}=\sum_{i} \mathrm{k}^{2} \mathrm{~m}_{i}\left(\frac{\vec{r}_{i}^{\prime}-\overrightarrow{\mathbf{r}}}{\rho_{i}^{3}}-\frac{\overrightarrow{\mathbf{r}}_{i}^{\prime}}{\mathbf{r}_{i}^{\prime 3}}\right)
$$

and $\vec{r}$ is the vector from Jupiter to the probe and $\vec{r}^{\prime}$ is from Jupiter to the disturbing body.

Equation (49) represents Cowell's equation of motion. When Encke's procedure is wanted the following is used:

$$
\frac{\mathrm{d}^{2} \delta \vec{r}}{\mathrm{dt}^{2}}=\frac{\mathrm{k}^{2} \mathrm{M}_{4}}{\mathrm{r}_{0}^{3}} \delta \vec{r}+\frac{\mathrm{k}^{2} \mathrm{~m}_{4}}{\mathrm{r}_{0}^{3}} \text { fq } \overrightarrow{\mathrm{r}}+\overrightarrow{\mathrm{F}}+\phi .
$$

Care must be taken in interpreting equations (48), (49) and (50) since $f$ and $q$ have different meanings depending on whether or not Encke's method is used.

\section{Integration of the Equations of Motion}

The above equations of motion either (49) or (50) are integrated over the time spent in the sphere of influence by means of a numerical integration technique (reference 17). This technique is a fourth order predictor-corrector with automatic adjustment of the time step of integration.

The integration is started at the time of the probe's entrance to the sphere of influence and is terminated at exit from the sphere. This termination is accomplished by checking the probe's distance from Jupiter until this distance becomes equal to the radius of the sphere. The integration is then halted and the resulting trajectory is determined. 
In the actual computer runs made, the use of the above Encke's equation (50) more than tripled the computer time when the moons of Jupiter were included as perturbing bodies. This increase in time was due in part to the greater number of calculations required and to the number of rectifications that occurred because of the inclusion of the moons. For this reason Cowell's method was used exclusively for the results included in this report. Encke's method was used as a check in only a few cases to insure that numerical accuracy was being retained: 


\section{THE GALILEAN SATELLITES OF JUPITER}

On the night of January 7, 1610 Galileo Galilei turned his crude telescope toward Jupiter and noticed three small "stars" in the vicinity of and in a straight line with the planet. Repeated observations showed that these "stars" moved with respect to Jupiter and on January 13 he saw four "stars". Further observations proved to him that he had indeed discovered satellites of Jupiter (reference 18).

The German astronomer Marius later independently discovered the Galilean moons and gave them their names (reference 19) as follows:

I Io

II Europa

III Ganymede

IV Callisto

Table III is a compilation of the physical data of these satellites and is taken in part from reference 20 .

When we consider that the planet Mercury has on the same scale a diameter of 0.38 , compared to 0.394 for Ganymede, we see that these moons are by no means small.

These four satellites have been found to be subject to very strong mutual perturbations which cause librations in their orbits about Jupiter. Laplace first discovered a resonance relationship between satellites I, II and III which shows that their mean motions are approximately in the proportion of 4:2:1 or in terms of mean longitudes $\mathrm{L}_{1}-3 \mathrm{~L}_{2}+2 \mathrm{~L}_{3}=180$ degrees (reference 21 ). If one 
Table III

The Galilean Satellites: Astronomical and Physical Data

\begin{tabular}{|l|c|c|c|c|}
\hline & $\mathrm{I}_{0}$ & Europa & Ganymede & Callisto \\
\hline Mass (Earth = 1) & 0.0121 & 0.0079 & 0.0261 & 0.0160 \\
Mean Diameter (Earth = 1) & 0.255 & 0.226 & 0.394 & 0.350 \\
Mean Density (H $\mathrm{H}_{2} \mathrm{O}=1$ ) & 4.03 & 3.78 & 2.35 & 2.06 \\
Surface Gravity (Earth = 1) & 0.19 & 0.16 & 0.17 & 0.13 \\
Velocity of Escape (km/sec) & 2.5 & 2.1 & 2.9 & 2.4 \\
Period of Axial Rotation (Days) & 1.77 & 3.55 & 7.15 & 16.69 \\
Mean Distance from Planet (km) & 421,400 & 670,500 & $1,069,500$ & $1,881,200$ \\
Sidereal Period (Days) & 1.769 & 3.551 & 7.155 & 16.689 \\
Mean Orbital Velocity (km/sec) & 17.37 & 13.77 & 10.90 & 8.22 \\
Radius of Sphere of Influence (km) & 7,200 & 9,600 & 24,800 & 36,100 \\
\hline
\end{tabular}

of the satellites is disturbed, the motions of the other two adjust so that this relationship is satisfied. Therefore the three satellites cannot be in conjunction or opposition at the same time (reference 22 ).

Little can be found in the literature on an accurate theory of the motions of the four moons but accurate positions are obviously necessary if a realistic investigation into their effects on the flyby probe is to be made. The theory of the moons is quite complex and interest in the theory has only recently begun to bring about useful investigations.

Sampson in 1910 (references 23 and 24) and De Sitter in 1931 (reference 25) have developed detailed theories to describe the motions of the Galilean satellites. Sampson's work is perhaps the best known and includes a rather extensive set of tables which are used to determine the position of the moons and to predict such physical phenomena as occulations and eclipses. This work which has become known as Sampson's Tables represents a monumental and 
complex task. Although difficult to use these tables give the times of phenomena to 0.000001 days and positions to $0^{\circ} .000001$ (reference 26). Andoyer (reference 27) somewhat simplified the procedure by using only the main terms of Sampson's tables and even though this is less accurate, Andoyer's procedure has been adopted by the Nautical Almanac Office in their publication of the phenomena of the moons.

More recently Ferraz Mello (reference 28) has studied the planar motion of the Galilean satellites in rectangular coordinates. He shows that the higher order harmonics of Jupiter's gravitational potential and the relativity corrections are negligable. Marsden (reference 29) has studied the motion of the four moons using Von Ziepel's method to eliminate the short period terms and to reduce the number of degrees of freedom.

The method used in this report to obtain the positions is a more or less simplified version of Sampson's method. The model used is one of a slowly moving plane common to all four satellites with some plane perturbations. This model leads to uncertainties in position of

$$
300 ; 6000 ; \quad 6000 ; \quad 25000 \quad \text { kilometers }
$$

respectively for satellites I, II, III and IV when compared with Sampson's Tables (reference 30). This method was received from Dr. Jean L. Sagnier in Paris, France who has spent much time investigating the theory of the moons of Jupiter.

A complete description of the information received from Dr. Sagnier appears in Appendix D and a complete and extensive discussion of Sampson's theory of the moons can be found in reference 23. The Tables in Appendix D are used to determine the positions of the moons which are in turn necessary to evaluate the disturbing acceleration in equation (39) above. This method of obtaining the positions of the moons has been programmed to generate an ephemeris of positions for each of the four moons. Such ephemerides do not seem to be easily available elsewhere. 
A comparison was made with examples worked out by Sampson in reference 20. Table IV shows the results of this comparison for satellites I and III on two different dates. The longitude is measured from the mean equinox of 1900 along the mean ecliptic to the ascending node of Jupiter's orbital plane and thereafter along this plane. The magnitude of the radius vector to the satellite is the projection of the radius vector into Jupiter's equatorial plane; and the column labeled "tan latitude" is the tangent of the elevation of the radius vector to Jupiter's orbital plane.

\section{Table IV}

Comparis on of Sagnier's Method and Sampson's Tables (Jovicentered)

\begin{tabular}{|c|r|c|c|}
\hline Satellite I June 2.156 1909 & Longitude & Radii (A. U.) & Tan Latitude \\
\hline \hline simplified method & $336^{\circ} .07021$ & 0.0000281304 & .018832 \\
Sampson's tables & $336^{\circ} .06863$ & 0.0000281296 & .0191815 \\
Idifferencel & $0^{\circ} .00158$ & $8 \times 10^{-10}$ & .0003 \\
\hline Satellite III April 3.841 1910 \\
\hline simplified method & $9^{\circ} .92885$ & 0.00714796 & .045659 \\
Sampson's tables & $9^{\circ} .92550$ & 0.00714794 & .045686 \\
Idifferencel & $0^{\circ} .00335$ & $2 \times 10^{-8}$ & .000027 \\
\hline
\end{tabular}




\section{ENCOUNTER GEOMETRIES AND MISSIONS USED IN THE STUDY}

Twenty different trajectories were studied to determine the effects of the Galilean moons. The launch date for injection into the transfer orbit from the Earth to Jupiter was March 8, 1972 which is one of the dates under consideration for a Galactic Jupiter Probe currently being planned by NASA (reference 31). Flight times of $450,500,550$ and 600 days from the Earth were used along with several combinations of the encounter parameters $B$ and $\Psi$. The first twelve cases included flight times of 450,500 and 550 days. The magnitude of the miss vector was held constant at $1.25 \times 10^{6} \mathrm{~km}$ and the angle $\Psi$ was varied from 0 to 360 degrees in steps of 90 degrees. The remaining 8 cases were obtained using a constant value of $180^{\circ}$ for $\Psi$ and values of $1.0 \times 10^{6}$ and $1.5 \times$ $10^{6} \mathrm{~km}$ for B . A flight time of 600 days was also included in these cases. These 8 cases represent the type of encounter geometry that is particularly useful in obtaining hyperbolic heliocentric trajectories which are capable of achieving far distances in relatively short times.

Some of the first twelve trajectories, particularly those where $\Psi=0^{\circ}$, are also useful for solar probes since they result in eccentricities of almost 1. These trajectories can in some cases impact the sun which cannot be done with direct flights from the Earth because of the energy required (references 8 and 11). However for these trajectories it is desirable that at exit from Jupiter's sphere of influence the probe be heading toward the sun, i.e. that the heliocentric true anomaly be greater than $180^{\circ}$. Otherwise the time required for the probe to reach the sun is increased prohibitively. 
Values of $90^{\circ}$ and $270^{\circ}$ for $\Psi$ are very useful in obtaining large distances both above and below the ecliptic plane and a little reflection will reveal that the two values ought to give symmetrical results (reference 8). Indeed it is possible to obtain a distance of 30 a.u. out of the ecliptic as did the case for 450 days where $\Psi=270^{\circ}$. However the time required to reach such distances will also be considerable and may rule out the possibility of such a flight being undertaken.

Other types of missions may also be studied if desired. These could include obtaining high inclinations of the orbit plane with the ecliptic, maximum velocity normal to the ecliptic and many other interesting orbits. Most of these would be difficult to attain in terms of the energy requirements without using the gravitational attraction of a large planet. This report includes only three types of mission objectives: the time required to reach 10 a.u. from the sun, the maximum distance attained out of the ecliptic, and close approaches to or impact with the sun. 


\section{RESULTS AND CONCLUSIONS}

The effects of the Galilean moons of Jupiter on the Jovian centered orbit are presented in Table $\mathrm{V}$. These results are presented as differences resulting from using only the sun as a disturbing body and then using the sun and moons as disturbing bodies. The first three columns identify the case with the parameters $B$ in kilometers, $\Psi$ in degrees and transfer time in days. The next six columns are the differences in the Keplerian elements as follows: the semi major axis (a), eccentricity (e), inclination (i), true anomaly ( $\theta$ ), right ascension of the ascending node $(\Omega)$, and arguement of perojove $(\omega)$. The next column is the difference in magnitude of the velocity at exit from Jupiter's sphere of influence. As described in a previous section the integration of the equations of motion is terminated when the probe reaches a distance of $48 \times 10^{6}$ kilometers from Jupiter which is the radius of the sphere of influence. Therefore, the magnitude of the radius vector is always the same at exit and differences can occur only in tangential position and in the velocity vector. The final four columns show the closest approach to the Galilean moons. The closest approaches to all four moons always occur within a span of 1.5 days and always near perijove of the approach trajectory where the probe is moving very fast.

A quick glance at the $\Delta a$ column shows that the only large differences occur in cases 11 and 19. The semi major axis is computed from

$$
a=\frac{r}{2-\frac{r v^{2}}{\mu}}
$$


and since $r$ is constant, the change in a is dependent only on $v$. Case 11 passes Europa by $6100 \mathrm{~km}$ and is the closest approach of all the cases. A close approach to one or more of the moons will of course perturb the trajectory. A study was made for case 11 in which one moon at a time was included into the equations of motion. Europa by itself caused about half of the perturbation and inclusion of Ganymede accounted for almost all of the remaining perturbation. However, Ganymede by itself accounted for little of the total perturbation. Hand calculations independent of the program substantiated the results obtained when a single moon was included. This study shows that a complex dynamical interaction between the bodies is occurring. This complex interaction cannot be predicted analytically in an easy way and therefore the perturbation is difficult to prove conclusively.

The change occurring in case 19 is even more difficult to explain. This trajectory does come closer to Ganymede than any other trajectory but is well outside Ganymede's sphere of influence (see table III). This case represents a 600 day transfer trajectory and as such is a relatively low energy Jupiter centered trajectory. Because of its low energy it is more sensitive to perturbing forces. As in case 11 approximate methods tended only to confirm the results obtained using one moon at a time. Again the explanation lies in the dynamical interaction between the bodies.

The other 18 cases do not exhibit any abnormally large changes in any of the elements.

It therefore appears from Table $\mathrm{V}$ that the moons of Jupiter do not represent any major perturbative force as far as the Jovian centered trajectory is concerned in a majority of the cases. This is consistent with what was originally thought when the problem was first undertaken. However, cases 11 and 19 indicate that under the right dynamical conditions, significant perturbations may result that will have to be considered. These perturbations cannot be predicted without including the moons. 
Table VI shows the resulting effects of the moons on the heliocentric trajectories at post encounter. The columns are the same as for Table $\mathrm{V}$ except a column has been added for the difference in the heliocentric radial distances.

Large differences occur in the semi major axis for the heliocentric orbits, which by virtue of the above equation are attributed to changes in the position and velocity magnitudes. Although these are large variations, it must be pointed out that the semi major axis is itself large. For example Case 12 shows a change in a of $.12 \times 10^{9} \mathrm{~km}$, where a equals $3 \times 10^{9} \mathrm{~km}$, representing a perturbation of about $4 \%$. This is the largest percentage of all 20 cases.

Cases 11 and 19 in the heliocentric orbits do not exhibit the large perturbations that occurred in the Jovicentric orbits. The variations in the semi major axis in the heliocentric cases are much less than $1 \%$. Indeed a glance at Table VI shows that none of the elements change very much and some exhibit smaller changes than the other 18 cases.

Table VII shows the differences resulting in the parameters that are of interest for a deep space post Jupiter encounter mission. The first three columns are the same as the previous tables. Columns 4 and 5 are found by taking the square root of the sum of the squares of the differences in components of the Jovicentric position and velocity vectors respectively. These numbers represent the straight line distance and velocity between the two types of perturbation runs. Column 6 is the difference in the time spent in Jupiter's sphere of influence. Columns 7, 8 and 9 are the respective differences in time to reach 10 a.u. from the sun; distances attained out of the ecliptic; and closest approach to the sun where these missions are applicable. Again the last 4 columns are a repetition of the close approaches to the moons.

Figure 10 (from reference 9) is a contour map of constant times to $10 \mathrm{~A} . \mathrm{U}$. This map was done for a 500 day transfer time and is in the $\vec{T}$ and $\vec{R}$ plane 
which is defined in a previous section. This plane is commonly called the impact plane and is perpendicular to the incoming asymptote. The angle $\Psi$ is measured around the perimeter of the figure and the magnitude of the miss vector (B) is measured by the concentric circles. A flight time of 600 days from post encounter to $10 \mathrm{~A} . \mathrm{U}$. can be achieved by any combination of the parameters B and $\Psi$ as obtained from the 600 day contour line. Anything inside this line represents a flight time of less than 600 days. The launch date for this figure was March 10, 1972 which is two days later than the launch date for Case 7 in Table V, but this difference will not alter Figure 10 by any appreciable amount. Figure 10 was drawn using only a two body determination as outlined in a previous section. Case 7 in Table VII took 558.990 days to reach $10 \mathrm{~A}$. U. when the sun and moons were included as perturbing bodies. The undisturbed time in Figure 10 was 556.528 days. From Table VII the time difference caused by the moons of Jupiter was 0.12 days. Cases 3 and 4 in Table VI may also be located on this contour map for $\mathrm{B}$ equal to $1.0 \times 10^{6}$ and $1.5 \times 10^{6} \mathrm{~km}$ respectively. Again it is seen that the influence of the moons is negligible. Also the difference between the perturbed and two body determination used in Figure 10 was less than four days so even the sun makes only a small difference as a disturbing body.

As far as the mission is concerned it is seen that the effects of the moons are very small indeed and can be neglected. However as far as determining the precise point of exit from the sphere of influence, columns 4 and 5 of Table VII clearly indicate that the moons do have an effect. These effects could be of critical importance depending on the particular post encounter mission being contemplated. This along with the changes in the heliocentric semi-major axis is sufficient to conclude that for purposes of orbit determination the Galilean moons must be included in any realistic study of a Jupiter Flyby. 


\begin{tabular}{|c|c|c|c|}
\hline \multirow{14}{*}{ 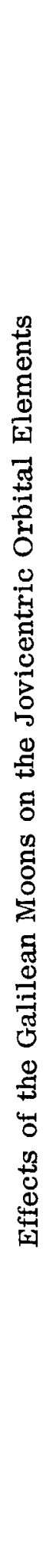 } & 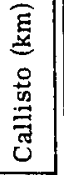 & 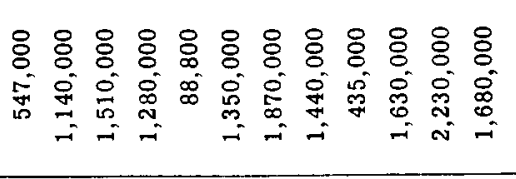 & 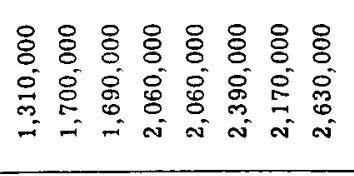 \\
\hline & 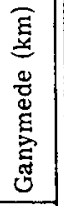 & 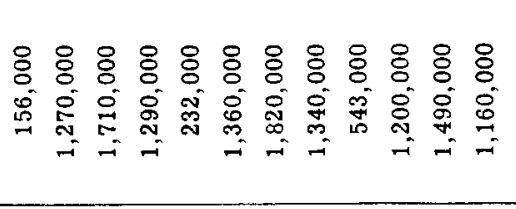 & 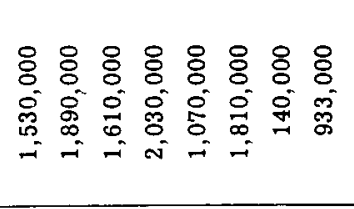 \\
\hline & 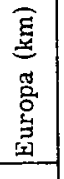 & 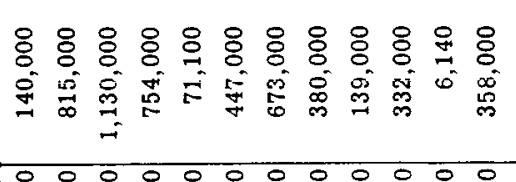 & 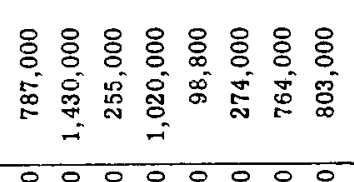 \\
\hline & 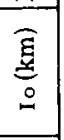 & 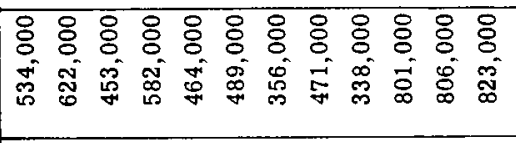 & 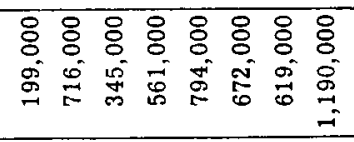 \\
\hline & 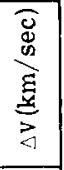 & 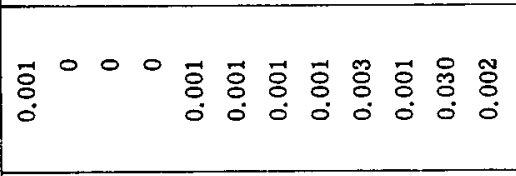 & $\begin{array}{lllllll}0 & \overrightarrow{0} & \overrightarrow{0} & \overline{0} & \overrightarrow{0} & \tilde{0} & 0 \\
0 & 0 & 0 \\
0 & 0 & 0 & 0 & 0 & 0 & 0 \\
0 & 0 & 0\end{array}$ \\
\hline & 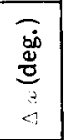 & 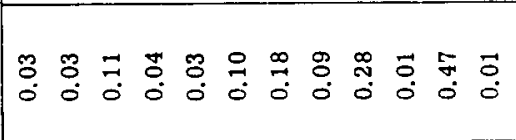 & 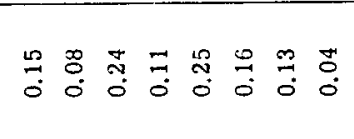 \\
\hline & 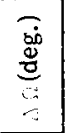 & 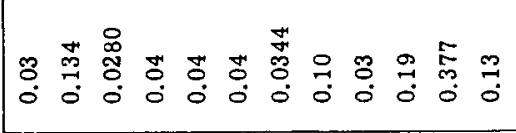 & 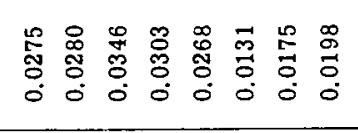 \\
\hline & 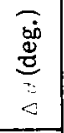 & 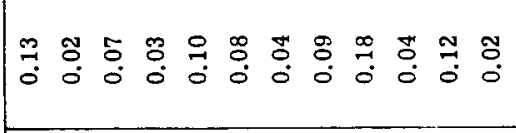 & 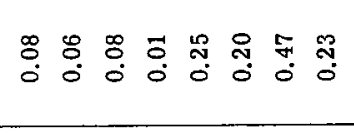 \\
\hline & $\frac{\vec{d}}{\stackrel{d}{\vec{d}}}$ & 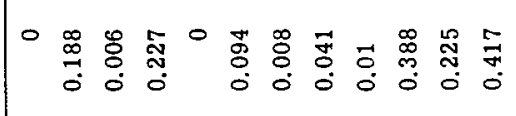 & 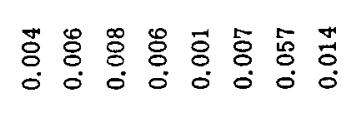 \\
\hline & $\therefore$ & 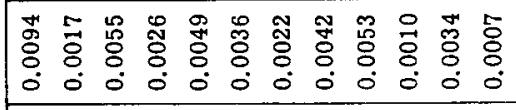 & 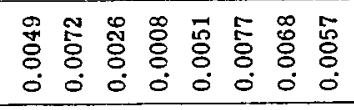 \\
\hline & 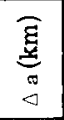 & 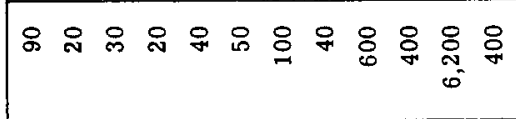 & "옹워 욤 8 \\
\hline & 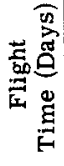 & 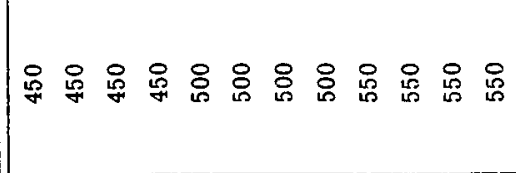 & 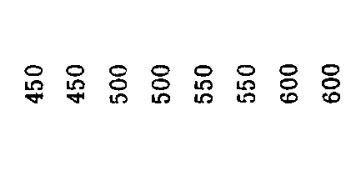 \\
\hline & $\begin{array}{l}\overline{80} \\
\frac{8}{0} \\
7\end{array}$ & ○ & 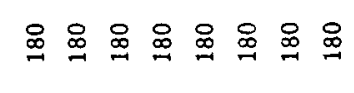 \\
\hline & 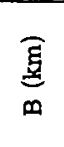 & 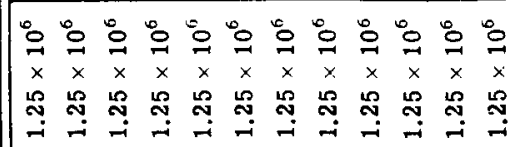 & 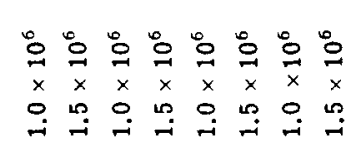 \\
\hline
\end{tabular}




\begin{tabular}{|c|c|c|c|}
\hline & 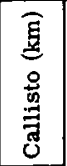 & 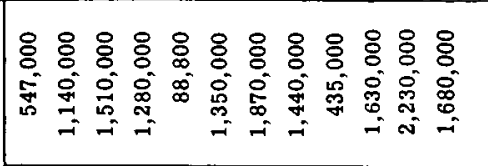 & 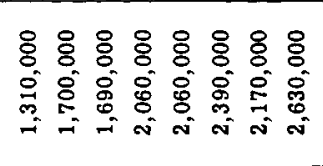 \\
\hline & 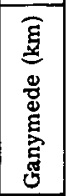 & 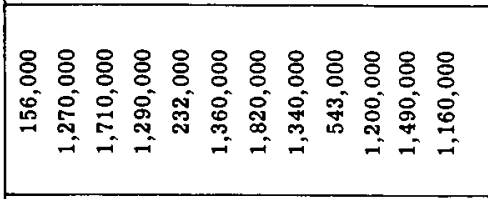 & 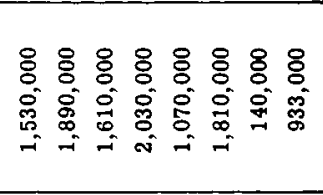 \\
\hline 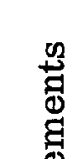 & 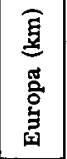 & 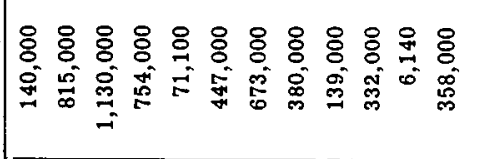 & 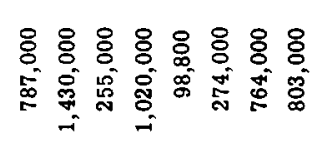 \\
\hline$\stackrel{0}{\rightarrow}$ & 言 & 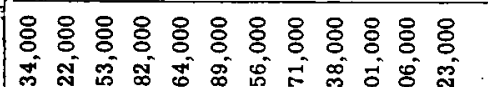 & 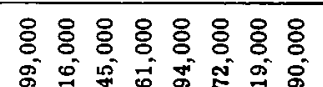 \\
\hline$\stackrel{\pi}{\stackrel{\pi}{\pi}}$ & 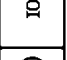 & 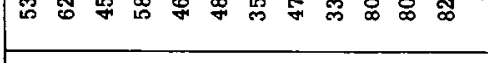 & बह \\
\hline 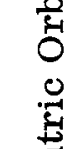 & 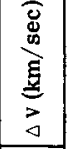 & 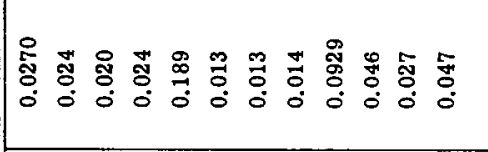 & 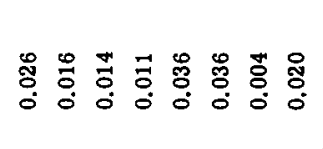 \\
\hline 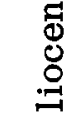 & $\underbrace{\frac{*}{\xi}}_{\frac{\alpha}{z}}$ & 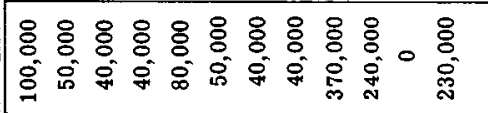 & 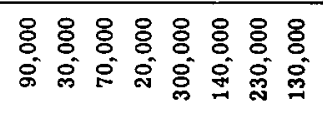 \\
\hline 量 & 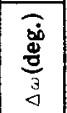 & 党 尽 & 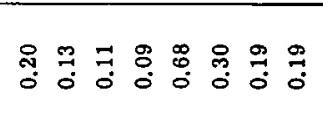 \\
\hline $\begin{array}{l}\text { g̊ } \\
\text { n }\end{array}$ & 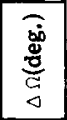 & 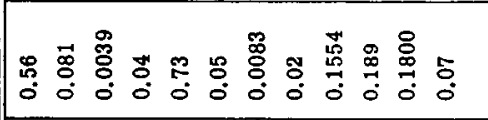 & 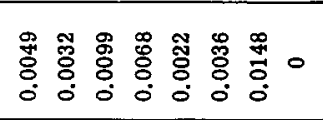 \\
\hline$\sum_{\substack{0\\
}}^{8}$ & 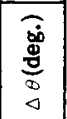 & 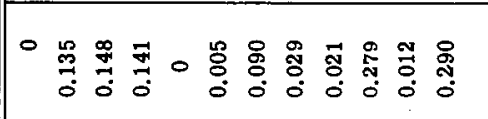 & 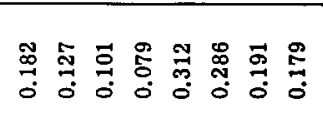 \\
\hline 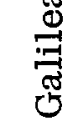 & 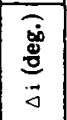 & 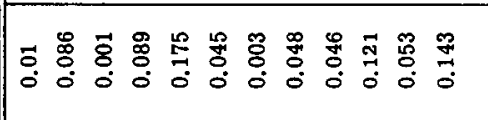 & 홍 \\
\hline$\underset{\ddagger}{\nsubseteq}$ & $\therefore$ & 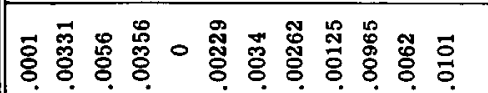 & 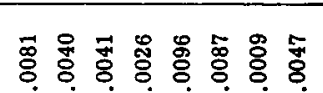 \\
\hline 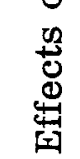 & $\begin{array}{l}\text { 蒠 } \\
\vdots \\
\triangleleft\end{array}$ & 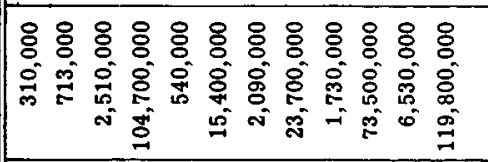 & 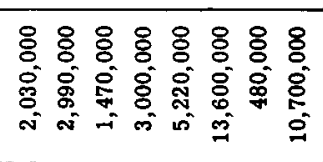 \\
\hline & 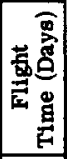 & 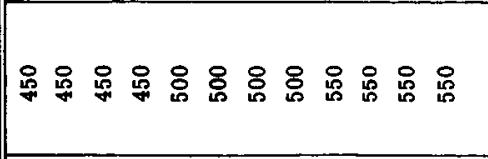 & 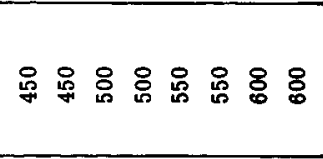 \\
\hline & 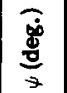 & $\circ 8 \%$ & 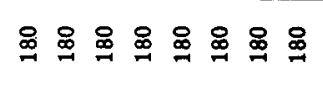 \\
\hline & $\sum_{\infty}^{\text {蒠 }}$ & 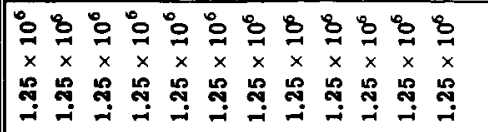 & 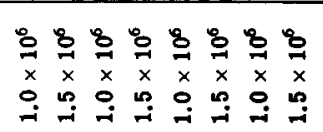 \\
\hline
\end{tabular}




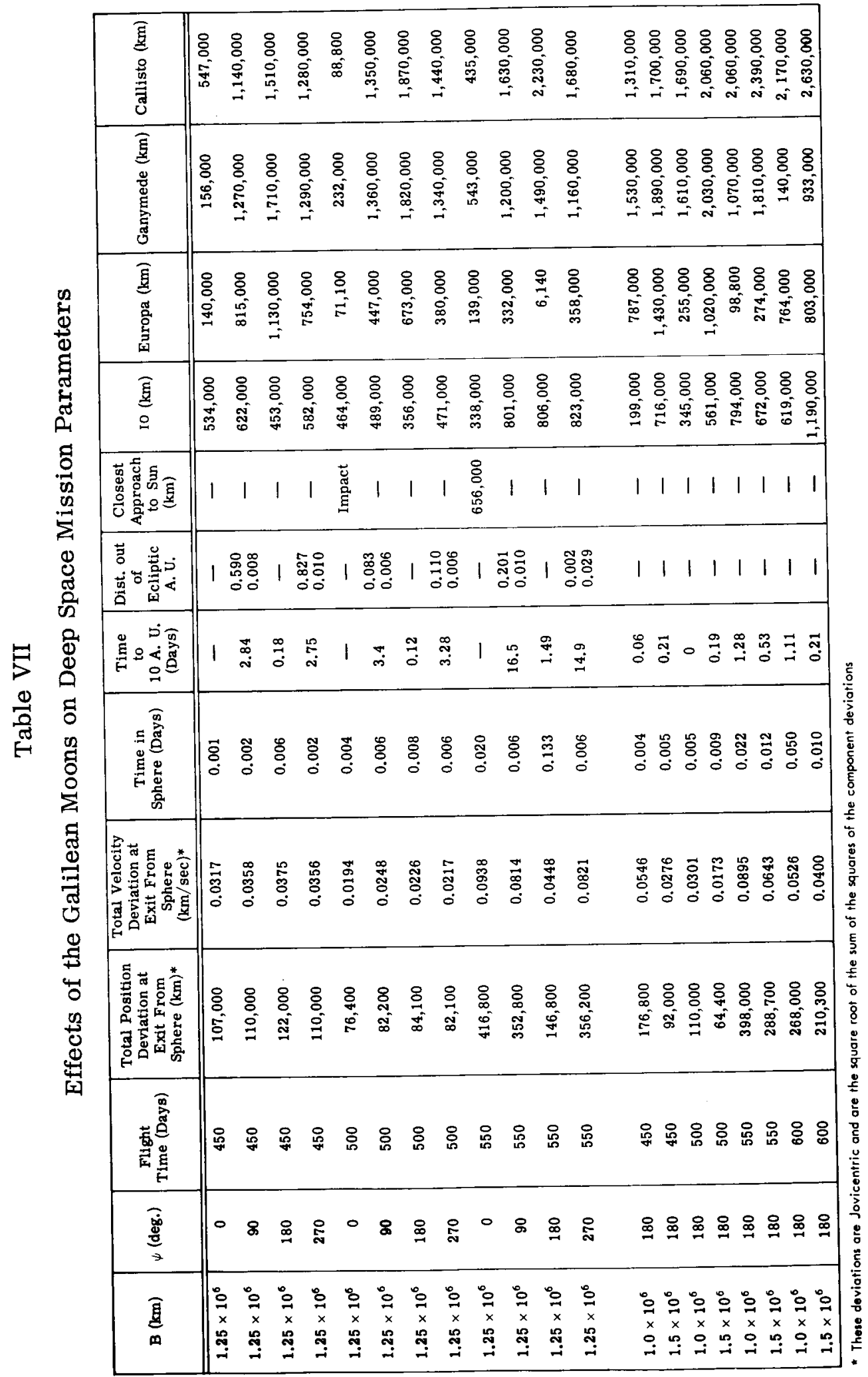




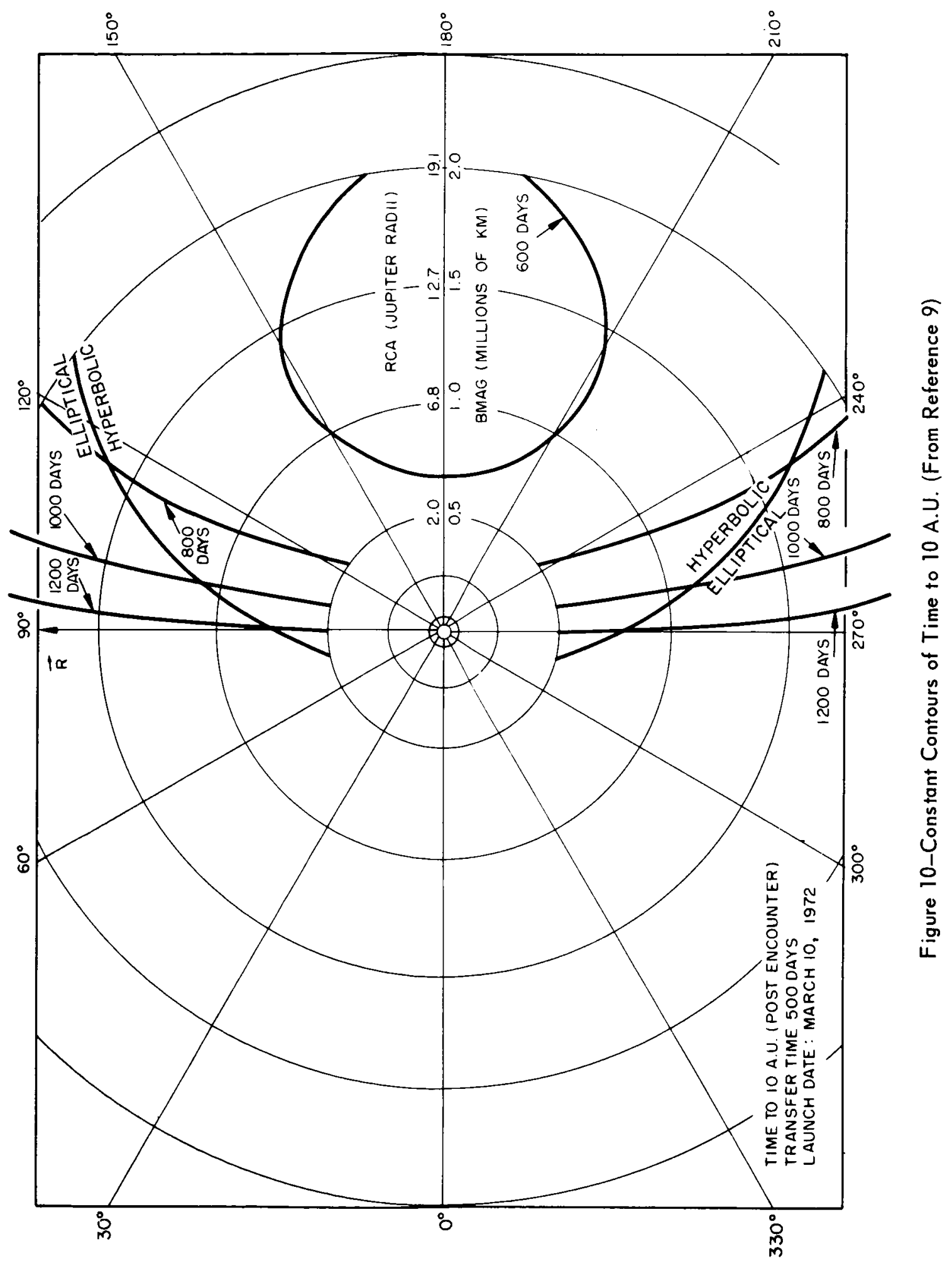




\section{SUMMARY}

The effects of the Galilean moons of Jupiter on a probe passing close to Jupiter were studied by using two different perturbative schemes. The first included only the sun as a perturbing body and the second included the sun and the four moons. Variations obtained from the two models are then the effects of the presence of the moons. An integrated trajectory was used only within Jupiter's sphere of influence with an approximate two body determination of the transfer trajectory between the Earth and Jupiter.

The numerical integration method was a predictor-corrector scheme with automatic adjustment of the step size and automatic error control. Due to considerable savings in machine time all cases were studied by integrating Cowell's equations of motion rather than Encke's equations.

While the total variations due to the Galilean moons in the Jovian centered orbital elements of the probe during the Jupiter flyby are fairly small, the actual point of exit on the sphere of influence is changed by an appreciable amount. Therefore as concerning gross trajectory determination one can essentially neglect these moons but when accurate position and velocity vectors are required, the perturbative effects of the moons most certainly should be included.

It is shown that the post encounter mission objectives such as time to reach 10 a.u. from the sun are also little changed due to the presence of the four larger moons of Jupiter. However the semi-major axis of the resulting heliocentric orbits do undergo large variations. The importance of the perturbations will 
depend on the exact nature of the post encounter missions that are to be studied such as a close approach to a second outer planet. Thus for accurate trajectory determination as well as accurate positions and velocities it is advisable to include the Galilean moons in the equations of motion. 


\section{APPENDIX A}

TO COMPUTE TIME FROM PERIAPSIS TO A GIVEN TRUE ANOMALY

This problem is easily solved using two body equations and the solution of Kepler's equation yields the desired result immediately.

Let: a be the semi-major axis

e the eccentricity

$\theta$ the true anomaly

$\mathrm{n}$ the mean motion

$\rho$ the semi latus rectum

$t$ the time from periapsis

$m$ the mean anomaly

E the eccentric anomaly

$r$ magnitude of radius vector

The relationship between $\theta$ and $E$ can be seen from figure A-1.

Now

$$
\begin{gathered}
\mathrm{X}=\xi-\mathrm{a}=\mathrm{a}(\cos \mathrm{E}-\mathrm{e})=\mathrm{r} \cos \theta \\
\mathrm{Y}=\eta=\mathrm{b} \sin \mathrm{E}=\mathrm{r} \sin \theta \\
\mathrm{b}=\mathrm{a} \sqrt{1-\mathrm{e}^{2}}
\end{gathered}
$$

also

$$
r=\frac{a\left(1-e^{2}\right)}{1+e \cos \theta}
$$

from $(A-1)$ and $(A-2)$ we obtain

$$
r=a(1-e \cos E)
$$


from $(A-1),(A-2)$, and $(A-4)$, we obtain

$$
\begin{aligned}
& \cos \theta=\frac{\cos E-e}{1-e \cos E} \\
& \sin \theta=\frac{\sqrt{1-e^{2}} \sin E}{1-e \cos E}
\end{aligned}
$$

and this allows us to finally obtain

$$
\operatorname{Tan} \frac{E}{2}=\sqrt{\frac{1-e}{1+e}} \operatorname{Tan} \frac{\theta}{2}
$$

The mean motion $\mathrm{n}$ is defined to be

$$
\mathrm{n}=\sqrt{\frac{\mu}{\mathrm{a}^{3}}}
$$

where $\mu$ is the gravitational constant of the central body. Using (A-5) to obtain E, Kepler's equation states that for an ellipse

Therefore

$$
M=E-e \sin E=n t
$$

$$
\mathrm{t}=\frac{\mathrm{M}}{\mathrm{n}}
$$

For an hyperbola, Kepler's equation becomes

$$
M=e \sinh E-E
$$

and again equation (A-7) holds.

For a parabola, a slightly different treatment is necessary. Equation (A-3) may be written as follows:

let $q=a(1-e)$

$$
r=\frac{a(1-e)(1+e)}{1+e \cos \theta}
$$

then upon setting $e=1$

Define

$$
r=\frac{2 q}{1+\cos \theta}
$$

then

$$
\mathrm{D}=\sqrt{2 \mathrm{q}} \mathrm{t} \text { an } \frac{\theta}{2}
$$

$$
r=\frac{1}{2}\left(2 q+D^{2}\right)
$$


The area using equation $(\mathrm{A}-10)$ is

$$
\begin{aligned}
A & =\frac{1}{2} \int_{0}^{\theta} r^{2} d \theta \\
& =\frac{1}{2} \sqrt{2 q}\left(q D+\frac{D^{3}}{6}\right)
\end{aligned}
$$

This yields

where

$$
\mathrm{t}=\frac{1}{\sqrt{\mu}}\left(\mathrm{qD}+\frac{\mathrm{D}^{3}}{6}\right)
$$

$$
\mathrm{n}=\sqrt{\mu}
$$

equation (A-11) may be found developed fully in reference 3. Equations (A-5), $(A-6),(A-7)$, and $(A-11)$ then allows one to compute the time for any type of orbit.

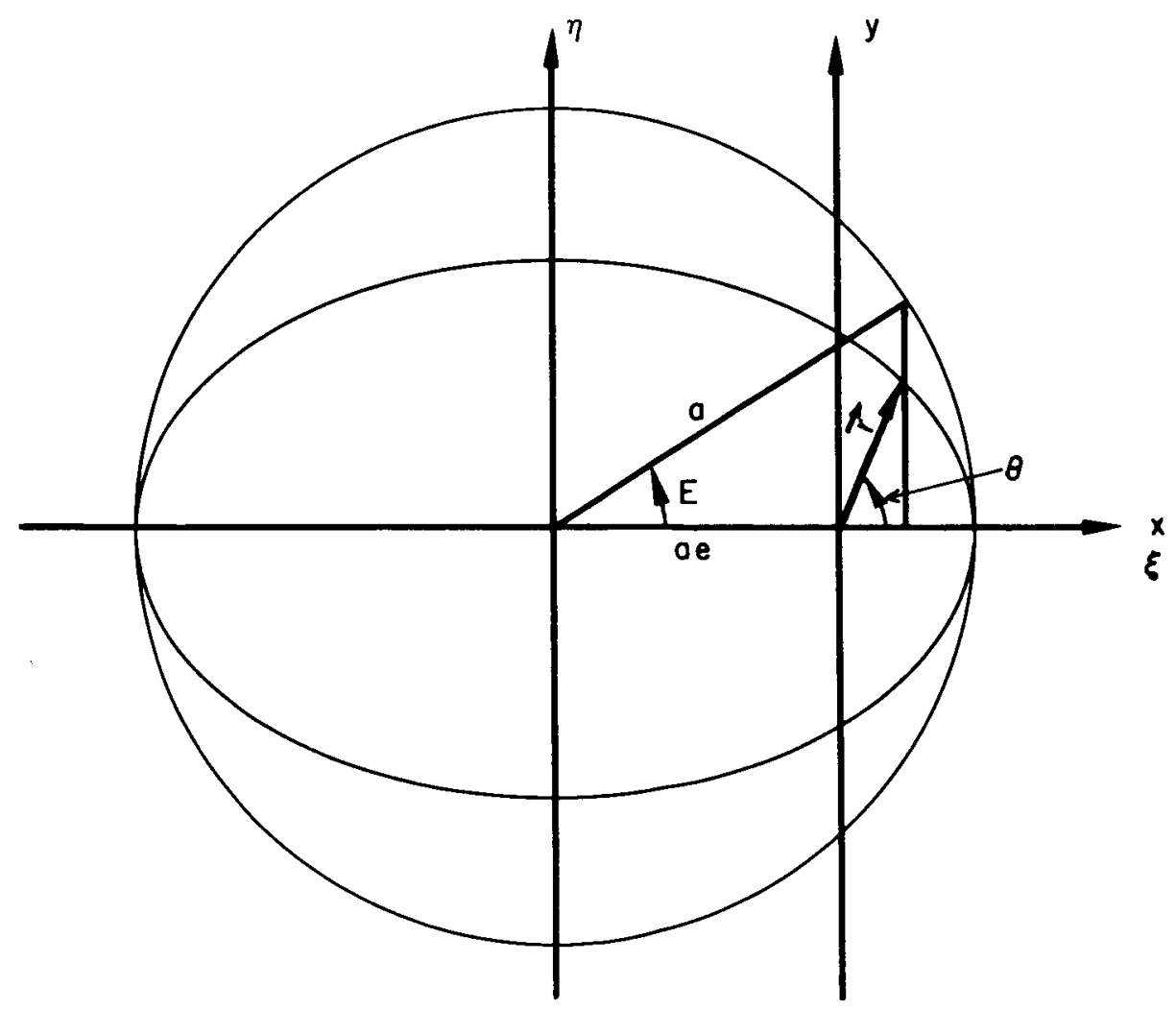

Figure A-1-Relationship Between True Anomaly and Eccentric Anomaly 


\section{APPENDIX B \\ TO UPDATE THE CARTESIAN COORDINATES OF THE PROBE USING AN INCREMENTAL TRUE ANOMALY}

The problem to be solved here is that of determining the position and velocity of a probe which is separated from a known position and velocity by an increment in the true anomaly. This treatment is essentially that found in reference 7 . The following notation will be used:

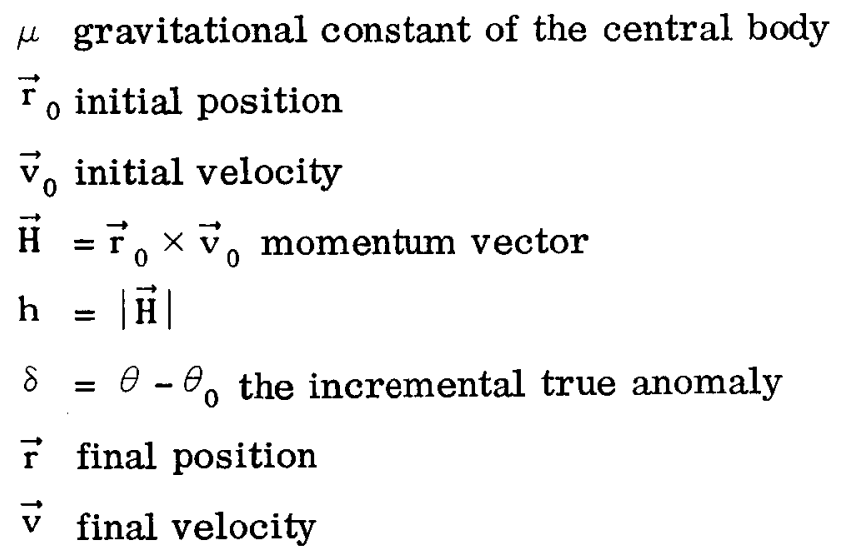

Since we are considering only two body planer motion we may write the following relationships:

$$
\left.\begin{array}{l}
\overrightarrow{\mathbf{r}}=\mathbf{f} \overrightarrow{\mathbf{r}}_{0}+\mathrm{g} \overrightarrow{\mathrm{v}}_{0} \\
\overrightarrow{\mathbf{v}}=\dot{\mathrm{f}} \overrightarrow{\mathbf{r}}_{0}+\dot{\mathrm{g}} \overrightarrow{\mathbf{v}}_{0}
\end{array}\right\}
$$

The quantities we wish to find are $f, g, \dot{f}$, and $\dot{g}$. 


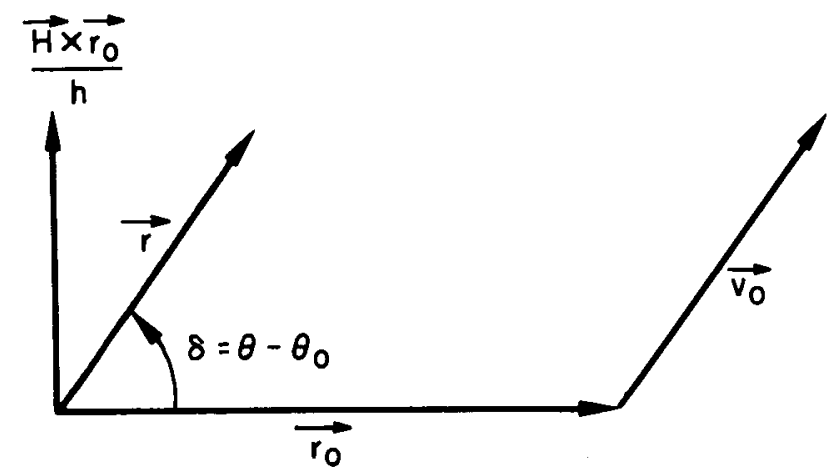

Figure B-1-Updating the Cartesian Coordinates

Figure B-1 will be helpful in understanding the geometry of the problem.

We first compute the momentum vector

$$
\overrightarrow{\mathrm{H}}=\overrightarrow{\mathrm{r}}_{0} \times \overrightarrow{\mathrm{v}}_{0}
$$

and observe that

$$
\frac{\overrightarrow{\mathrm{H}} \times \overrightarrow{\mathrm{r}}_{0}}{\mathrm{~h}}
$$

has magnitude equal to $\mathrm{r}_{0}$.

From figure B-1 we can decompose $\vec{r}$ onto $\vec{r}_{0}$ and $\frac{\vec{H}_{0} \times \vec{r}_{0}}{h}$ and obtain respectively $r \cos \delta$ and $r \sin \delta$.

We may therefore write for $\vec{r}$

$$
\vec{r}=\frac{r}{r_{0}}\left[\vec{r}_{0} \cos \delta+\frac{\vec{H} \times \vec{r}_{0}}{h} \sin \delta\right]
$$

and

$$
\overrightarrow{\mathrm{H}} \times \overrightarrow{\mathrm{r}}_{0}=\left(\overrightarrow{\mathrm{r}}_{0} \times \overrightarrow{\mathrm{v}}_{0}\right) \times \overrightarrow{\mathrm{r}}_{0}=\overrightarrow{\mathrm{v}}_{0}\left(\overrightarrow{\mathrm{r}}_{0} \cdot \overrightarrow{\mathrm{r}}_{0}\right)-\overrightarrow{\mathrm{r}}_{0}\left(\overrightarrow{\mathrm{r}}_{0} \cdot \overrightarrow{\mathrm{v}}_{0}\right)
$$

then equation (B-2) becomes

$$
\overrightarrow{\mathbf{r}}=\frac{\mathbf{r}}{\mathbf{r}_{0}}\left[\vec{r}_{0}\left(\cos \delta-\frac{\vec{r}_{0} \cdot \vec{v}_{0}}{h} \sin \delta\right)+\vec{v}_{0} \frac{\mathrm{r}_{0}^{2}}{h} \sin \delta\right]
$$


Comparing this with equation $(B-1)$, we see that

$$
\begin{aligned}
& f=\frac{r}{r_{0}}\left(\cos \delta-\frac{\vec{r}_{0} \cdot \vec{v}_{0}}{h} \text { in } \delta\right) \\
& g=\frac{r r_{0}}{h} \sin \delta
\end{aligned}
$$

However, the magnitude $r$ in the above equation is still unknown. To solve for $r$ we make use of the radius equation

$$
r=\frac{a\left(1-e^{2}\right)}{1+e \cos \theta}=\frac{\rho}{1+e \cos \theta}
$$

and

$$
\mathrm{a}\left(1-\mathrm{e}^{2}\right)=\frac{\mathrm{h}^{2}}{\mu}
$$

Thus

$$
r=\frac{h^{2}}{\mu(1+e \cos \theta)}
$$

also

$$
\theta=\delta+\theta_{0}
$$

Therefore

$$
\cos \theta=\cos \delta \cos \theta_{0}-\sin \delta \sin \theta_{0}
$$

Using equations (B-5) and (B-6), we immediately obtain

$$
\cos \theta_{0}=\frac{h^{2}-\mu r_{0}}{\mu r_{0} e}
$$

Note that from the radius equation we may write

and by using

$$
\begin{aligned}
\dot{\mathrm{r}}_{0}=\frac{\mathrm{d}}{\mathrm{dt}}\left(\overrightarrow{\mathrm{r}}_{0} \cdot \overrightarrow{\mathbf{r}}_{0}\right)^{1 / 2} & =\frac{\overrightarrow{\mathrm{r}}_{0} \cdot \overrightarrow{\mathrm{v}}_{0}}{\mathbf{r}_{0}} \\
& =\frac{e \rho \dot{\theta} \sin \theta_{0}}{\left(1+e \cos \theta_{0}\right)^{2}}
\end{aligned}
$$

$$
\mathrm{h}=\mathrm{r}_{0}^{2} \dot{\theta}_{0}
$$


we obtain

$$
\sin \theta_{0}=\frac{h \dot{r}_{0}}{\mu e}
$$

from above

$$
\dot{r}_{0}=\frac{\vec{r}_{0} \cdot \vec{v}_{0}}{r_{0}}
$$

which together with equation (B-8) gives

$$
\sin \theta_{0}=\frac{h \vec{r}_{0} \cdot \vec{v}_{0}}{r_{0} \mu e}
$$

using equations (B-5), (B-6), (B-7), and (B-10) we obtain

$$
r=\frac{\frac{h^{2}}{\mu}}{1+e\left[\frac{\cos \delta\left(h^{2}-\mu r_{0}\right)}{\mu r_{0} e}-\sin \delta \sin \theta_{0}\right]}
$$

or

$$
\frac{r}{r_{0}}=\frac{h^{2}}{\mu r_{0}+\left(h^{2}-\mu r_{0}\right) \cos \delta-h\left(\vec{r}_{0} \cdot \vec{v}_{0}\right) \sin \delta}
$$

Equation (B-11) allows the computation of $f$ and $g$ in equation (B-4) above. In an entirely similar manner we may also obtain $\dot{\mathrm{f}}$ and $\dot{\mathrm{g}}$ :

$$
\left.\begin{array}{l}
\dot{\mathrm{f}}=\frac{\mu}{\mathrm{r}_{0} \mathrm{~h}^{2}}\left[\left(\overrightarrow{\mathrm{r}}_{0} \cdot \overrightarrow{\mathrm{v}}_{0}\right)(1-\cos \delta)-\mathrm{h} \sin \delta\right] \\
\dot{\mathrm{g}}=1-\frac{\mu \mathrm{r}_{0}}{\mathrm{~h}}(1-\cos \delta)
\end{array}\right\}
$$

We may gain as a dividend the time difference corresponding to $\delta$ in the following manner:

$$
\begin{gathered}
a=\frac{r_{0}}{\left(2-\frac{r_{0} v^{2}}{\mu}\right)} \\
\rho=\frac{h^{2}}{\mu}=a\left(1-e^{2}\right)
\end{gathered}
$$


which yields

$$
\mathrm{e}^{2}=1-\frac{\rho}{\mathrm{a}}
$$

From equations (B-7) and B-10) for $\theta$ we obtain

$$
\operatorname{Tan} \theta=\frac{\mathrm{h}(\overrightarrow{\mathrm{r}} \cdot \overrightarrow{\mathbf{v}})}{\mathrm{h}^{2}-\mu \mathrm{r}}
$$

then

$$
\theta=\theta_{0}+\delta
$$

and the method of Appendix A will yield the time difference between $\theta_{0}$ and $\theta$. 


\section{APPENDIX C}

TO FIND $\vec{r}$ AND $\vec{v}$ OF A PROBE ON AN HYPERBOLIC ORBIT SEPARATED FROM $\vec{r}_{0}$ AND $\vec{v}_{0}$ BY TIME $\triangle t$

The following method is used to step along an hyperbolic orbit knowing an initial position and velocity $\overrightarrow{\mathrm{r}}_{0}$ and $\overrightarrow{\mathrm{v}}_{0}$ and a time difference $\Delta \mathrm{t}$.

Reference to figure C-1 shows the hyperbola and the geometry of the problem to be solved. The axis $\vec{i}_{\xi}$ and $\vec{i}_{\eta}$ are unit vectors, origin at the focus and such that they form a right handed system.

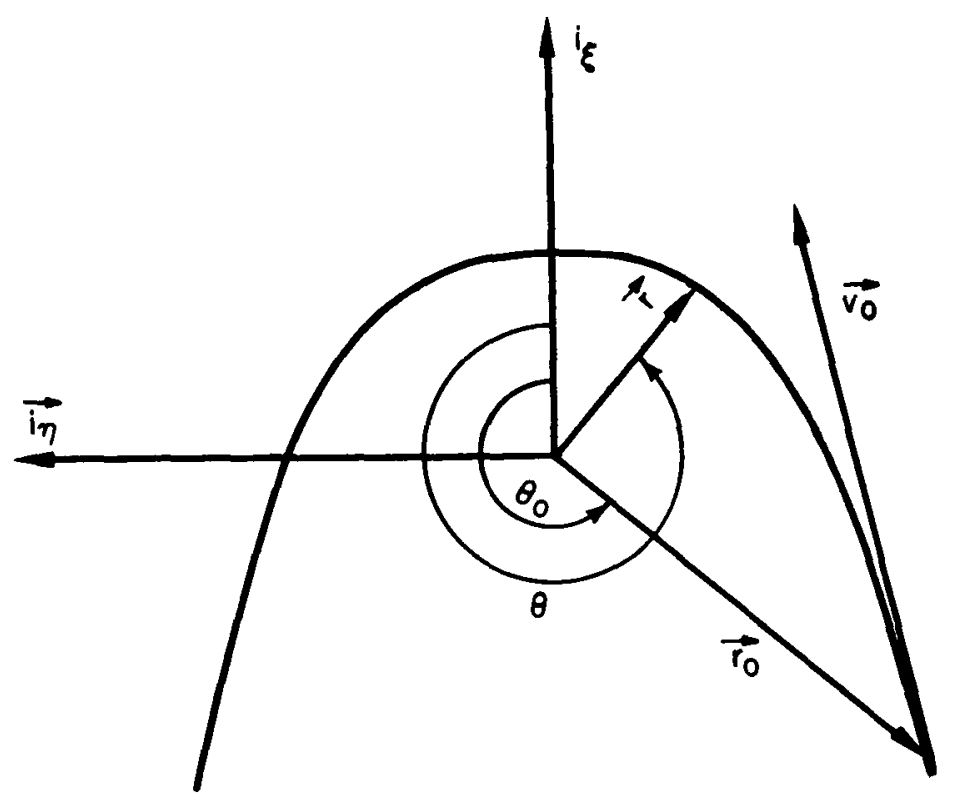

Figure C-1-The Hyperbolic Orbit Geometry 
From figure $\mathrm{C}-1$ it is obvious that

$$
\vec{r}=r \cos \theta \vec{i}_{\xi}+r \sin \theta \vec{i}_{\eta}
$$

and the well known relationships for a hyperbola are:

$$
\begin{aligned}
& r=a(e \cosh F-1) \\
& \cos \theta=\frac{e-\cosh F}{e \cosh F-1} \\
& \sin \theta=\frac{\sqrt{e^{2}-1} \sinh F}{e \cosh F-1}
\end{aligned}
$$

where $\mathrm{F}$ is the "hyperbolic" eccentric anomaly. Upon substituting $(\mathrm{C}-2),(\mathrm{C}-3)$, and $(\mathrm{C}-4)$ into $(\mathrm{C}-1)$, we obtain

$$
\vec{r}=a(e-\cosh F) \vec{i} \xi+\sqrt{a \rho} \sinh F \vec{i}_{\eta}
$$

where $\rho$ is the semi latus rectum. Differentiating equation (C-5) and noting that from Kepler's equation

$$
\frac{\mathrm{dF}}{\mathrm{dt}}=\sqrt{\frac{\mu}{\mathrm{a}^{3}}} \frac{1}{\mathrm{e} \cosh \mathrm{H}-1}
$$

we obtain

$$
\overrightarrow{\mathbf{v}}=-\frac{\sqrt{\mu \mathrm{a}}}{\mathbf{r}} \sinh \mathrm{F} \overrightarrow{\mathrm{i}}_{\xi}+\frac{\sqrt{\mu \rho}}{\mathrm{r}} \cosh \mathrm{F} \overrightarrow{\mathrm{i}}_{\eta}
$$

Equations (C-5) and (C-6) allow the computation of position and velocity anywhere along the hyperbola if the parameter $F$ is known since $a, e$, and $\rho$ may be easily calculated from $\vec{r}_{0}$ and $\vec{v}_{0}$. We will use $\vec{r}_{0}$ and $\vec{v}_{0}$ to calculate $F$ and to determine the axis $\vec{i}_{\xi}$ and $\vec{i}_{\eta}$.

From the Laplace Integral of two body motion we have

$$
\overrightarrow{\mathbf{E}}=\frac{1}{\mu} \overrightarrow{\mathbf{v}}_{0} \times \overrightarrow{\mathbf{c}}_{0}-\overrightarrow{\mathbf{r}}_{0}^{\circ}
$$

where $\vec{E}$ is a vector in the direction of perihelion and

$$
\vec{c}_{0}=\vec{r}_{0} \times \vec{v}_{0}
$$


Obviously

$$
\overrightarrow{\mathrm{i}}_{\xi}=\frac{\overrightarrow{\mathrm{E}}}{|\overrightarrow{\mathrm{E}}|}
$$

also

$$
\overrightarrow{\mathrm{i}}_{\eta}=\frac{\overrightarrow{\mathrm{c}}_{0} \times \overrightarrow{\mathrm{E}}}{\left|\overrightarrow{\mathrm{c}}_{0}\right||\overrightarrow{\mathrm{E}}|}
$$

From the energy equation we have

$$
a=\frac{\mu r_{0}}{2 \mu-r_{0} v_{0}^{2}}
$$

then

$$
\rho=\mathbf{a}\left|\left(\mathrm{e}^{2}-1\right)\right|
$$

where

$$
e=|\vec{E}|
$$

The mean motion is then

$$
\mathrm{n}=\sqrt{\frac{\mu}{\mathrm{a}^{3}}}
$$

and the true anomaly at the initial time is

$$
\theta_{0}=\cos ^{-1}\left(\frac{\rho-\mathrm{r}_{0}}{\mathrm{r}_{0} \mathrm{e}}\right)
$$

The change in the "hyperbolic" mean anomaly is

$$
\Delta \mathrm{M}=\mathrm{n} \Delta \mathrm{t}
$$

The value for $F_{0}$ the eccentric anomaly at the initial point is obtained from

$$
\sinh F_{0}=\frac{\sqrt{e^{2}-1} \sin \theta_{0}}{e \cos \theta_{0}+1}
$$

Then the value of the mean anomaly $(M)$ at the point $\vec{r}$ is obtained by adding equation $(C-10)$ to Kepler's equation evaluated at $F_{0}$

$$
M=e \sinh F_{0}-F_{0}+\Delta M
$$

or

$$
M=e \sinh F-F
$$


where $F$ is the unknown eccentric anomaly needed in equations (C-5) and (C-6). Equation $(\mathrm{C}-11)$ can be solved for $\mathrm{F}$ by an interative procedure as follows:

we form the difference function

$$
\Delta_{n}=M-e \sinh F_{n}+F_{n}
$$

and as a first guess we choose $F_{1}=0$. Using the Newton interative formula we have

$$
F_{n+1}=F_{n}-\frac{\Delta_{n}}{\Delta_{n}^{\prime}}
$$

or

$$
F_{n+1}=F_{n}-\frac{\left(M-e \sinh F_{n}+F_{n}\right)}{1-e \cosh F_{n}}
$$

when $\Delta_{n}$ becomes small enough we have the required value for $F$ and $\vec{r}$ and $\vec{v}$ may now be evaluated. 


\author{
APPENDIX D \\ Received from Dr. J. L. Sagnier, Paris, France \\ THE POSITIONS OF JUPITER'S GREAT SATELLITES
}

The following tables include:

- The initial values and daily motions of the arguments occurring in the subsequent series, expressed in radians when taking into account the factor $2 \pi$.

- The series defining the mean positions of Jupiter's equatorial plane and of the orbital planes of the satellites.

- The equatorial true longitudes of the satellites, in radians from Sampson's $\mathrm{x}$-axis up to the ascending node of Jupiter's equator on Sampson's $(x, y)$ - plane, and thereafter along Jupiter's equator.

- The projected values on Jupiter's equator of the radii vectors, expressed in A. U.

- The reductions to Sampson's $(\mathrm{x}, \mathrm{y})$ - plane, to be added to the equatorial true longitudes, when the longitudes in Sampson's system are needed.

- The tangents of the latitudes on Sampson's $(x, y)$-plane.

The unit of time is always the ephemeris day, initial epochs are explicitly given when necessary. The unit of angle is the radian, except for some phase angles in the arguments expressed in degrees. The unit of length is the astronomical unit. 
A low accuracy determination of the positions of Jupiter's satellites may be conveniently obtained by the introduction of the most significant terms of the radii vectors and of the equatorial longitudes in the mean orbital planes, whether approximated by the Jovian equator or not. But the use of the series reducing longitudes to Sampson's system and of those giving the latitudes in the same, is necessary for a full accuracy computation.

Arguments of Series

\begin{tabular}{|c|c|c|c|c|c|c|c|c|}
\hline \multirow{2}{*}{$\frac{\operatorname{Arg}^{\mathrm{t}}}{\ell_{1}}$} & \multicolumn{4}{|c|}{ Value for $0^{\text {h }} \mathrm{TE}, 31 / 12 / 1959=2 \pi \times$} & \multicolumn{4}{|c|}{ Daily variation $=2 \pi \times$} \\
\hline & 0.9406 & 5442 & 8812 & 5000 & +0.5652 & 4720 & 1208 & 3333 \\
\hline$\ell_{2}$ & 0.0427 & 5197 & 0477 & 7778 & +0.2815 & 9656 & 0200 & 0000 \\
\hline$\ell_{3}$ & 0.3438 & 0071 & 0875 & 0000 & +0.1397 & 7123 & 9694 & 4444 \\
\hline$\ell_{4}$ & 0.7025 & 5215 & 8347 & 2222 & +0.0599 & 1974 & 8972 & 2222 \\
\hline$\pi 1$ & 0.3517 & 7383 & 1250 & 0000 & +0.0004 & 3870 & 9722 & 2222 \\
\hline$\pi_{2}$ & 0.4138 & 9361 & 2777 & 7778 & +0.0001 & 3087 & 6666 & 6667 \\
\hline$\pi_{3}$ & 0.3694 & 6197 & 9305 & 5556 & +0.0000 & 1930 & 9166 & 6667 \\
\hline$\pi_{4}$ & 0.9023 & 3018 & 4027 & 7778 & +0.0000 & 0527 & 0833 & 3333 \\
\hline$\omega_{1}$ & 0.9339 & 4899 & 5138 & 8889 & -0.0003 & 7230 & 6944 & 4444 \\
\hline$\omega_{2}$ & 0.8166 & 4930 & 4027 & 7778 & -0.0000 & 9083 & 1388 & 8889 \\
\hline$\omega_{3}$ & 0.4661 & 1489 & 5555 & 5556 & -0.0000 & 1938 & 2222 & 2222 \\
\hline$\omega_{4}$ & 0.9135 & 1122 & 8055 & 5556 & -0.0000 & 0487 & 6111 & 1111 \\
\hline$\psi$ & 0.8801 & 0470 & 7361 & 1111 & +0.0000 & 0009 & 9722 & 2222 \\
\hline $\mathrm{G}$ & 0.6840 & 6586 & 4444 & 4444 & +0.0002 & 3080 & 8888 & 8889 \\
\hline $\mathrm{G}$ & 0.5249 & 4424 & 2500 & 0000 & +0.0000 & 9294 & 3888 & 8889 \\
\hline$\Pi$ & 0.0392 & 7971 & 0277 & 7778 & +0.0000 & 0010 & 6111 & 1111 \\
\hline
\end{tabular}

For accurate computations, argument $\mathrm{G}$ should be supplemented in each of its occurrences by the long period correction:

$$
\begin{aligned}
2 \pi & {\left[0.95000000 \times 10^{-4} \mathrm{~s} \text { in } 2 \pi\left(0.36965917-0.44921389 \times 10^{-4}[\mathrm{t}-1960.0]_{\mathrm{days}}\right)\right.} \\
+ & \left.0.92083333 \times 10^{-3} \mathrm{sin} 2 \pi\left(0.43614306+0.29277778 \times 10^{-5}[\mathrm{t}-1960.0]_{\mathrm{days}}\right)\right]
\end{aligned}
$$


Positions of Equatorial and Orbital Planes

$\xi_{\mathrm{i}}, \eta_{\mathrm{i}}$ : In Sampson's coordinate system, the first two components of a vector of modulus 1 , perpendicular to the mean orbital plane of the $i^{\text {th }}$ satellite (for $i=1,2,3,4$ ) or Jupiter's equatorial plane (for $i=0$ ).

$$
\xi_{i} \times 10^{6}=\Sigma \mathrm{A} \sin \alpha, \eta_{i} \times 10^{6}=\Sigma(-\mathrm{A}) \cos \alpha
$$

the coefficients $\mathrm{A}$ and the arguments $\alpha$ being:

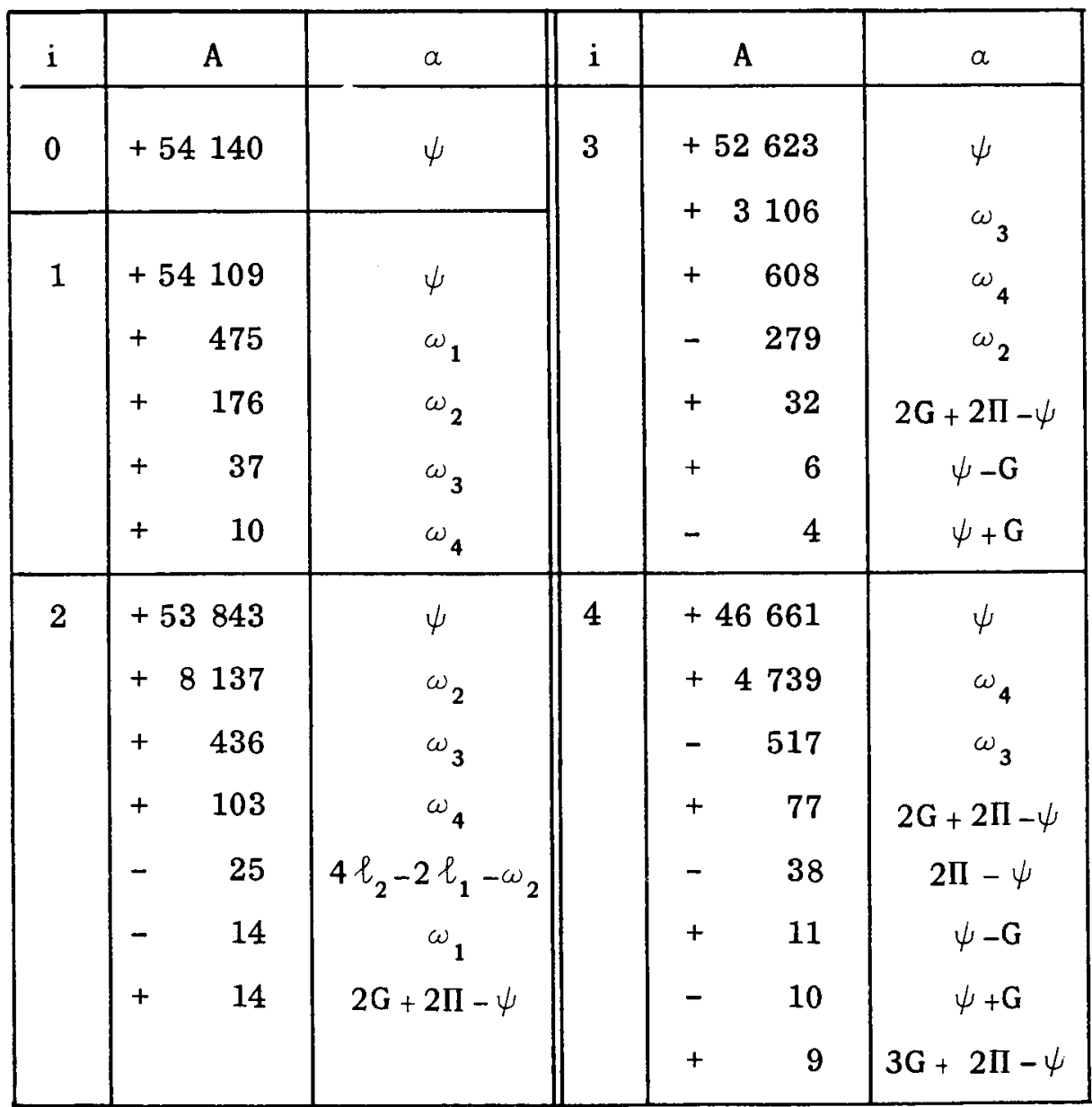


Equatorial True Longitudes

For the $i^{\text {th }}$ satellite, we have:

$$
\mathbf{v}_{i}=\ell_{i}+2 \pi \times 10^{-8} \cdot \sum A \sin \alpha
$$

with the following values for $A$ and expressions for $\alpha$ :

\begin{tabular}{|c|c|c|c|c|c|c|}
\hline & A & $\alpha$ & A & $a$ & A & $\alpha$ \\
\hline \multicolumn{7}{|c|}{ FIRST SATELLITE } \\
\hline- & 1097 & $\ell_{1}-\ell_{2}$ & $+\quad 39$ & $3 l_{1}-3 l_{3}$ & -431 & G \\
\hline+1 & 30978 & $2 \ell_{1}-2 \ell_{2}$ & + & $4 l_{1}-4 l_{3}$ & $+\quad 97$ & $2 \mathrm{G}$ \\
\hline+ & 439 & $3 l_{1}-3 l_{2}$ & +1478 & $\ell_{1}-\pi_{1}$ & -206 & $\psi-\omega_{1}$ \\
\hline+ & 431 & $4 \ell_{1}-4 \ell_{2}$ & +1183 & $\ell_{1}-\pi_{3}$ & +56 & $\psi-\omega_{2}$ \\
\hline+ & 33 & $5 \ell_{1}-5 \ell_{2}$ & +528 & $l_{1}-\pi_{4}$ & +214 & $\psi-\omega_{2}$ \\
\hline+ & 14 & $6 l_{1}-6 l_{2}$ & -2000 & $\ell_{2}-2 \ell_{3}+\pi_{1}$ & $-\quad 97$ & $\psi-\omega_{4}$ \\
\hline+ & 6 & $7 l_{1}-7 l_{2}$ & +1094 & $\ell_{2}-2 l_{3}+\pi_{2}$ & +267 & $\omega_{2}-\omega_{3}$ \\
\hline- & 439 & $l_{1}-l_{3}$ & +2517 & $\ell_{2}-2 \ell_{3}+\pi_{3}$ & & \\
\hline+ & 369 & $2 l_{1}-2 l_{3}$ & +983 & $\ell_{2}-2 \ell_{3}+\pi_{4}$ & & \\
\hline \multicolumn{7}{|c|}{ SECOND SATELLITE } \\
\hline & 3283 & $l_{2}-l_{3}$ & +2625 & $l_{2}-\pi_{2}$ & +94 & $2 \ell_{3}-2 \mathrm{G}-2 \mathrm{II}$ \\
\hline & 97267 & $2 \ell_{2}-2 \ell_{3}$ & +9794 & $\ell_{2}-\pi_{3}$ & -2908 & G \\
\hline+ & 1333 & $3 l_{2}-3 l_{3}$ & +4119 & $l_{2}-\pi_{4}$ & -325 & $2 \mathrm{G}$ \\
\hline+ & 1503 & $4 \ell_{2}-4 \ell_{3}$ & +3169 & $\ell_{2}-2 l_{3}+\pi_{1}$ & -236 & $5 \mathrm{G}^{\prime}-2 \mathrm{G}+48^{\circ} \cdot 6$ \\
\hline+ & 106 & $5 \ell_{2}-5 l_{3}$ & +2125 & $\ell_{2}-2 \ell_{3}+\pi_{2}$ & +128 & $\pi_{3}-\pi_{4}$ \\
\hline+ & 150 & $6 l_{2}-6 l_{3}$ & -12375 & $\ell_{2}-2 \ell_{3}+\pi_{3} \mid$ & -3514 & $\psi-\omega_{2}$ \\
\hline+ & 19 & $7 \ell_{2}-7 \ell_{3}$ & -4994 & $l_{2}-2 l_{3}+\pi_{4}$ & -544 & $\psi-\omega_{3}$ \\
\hline- & 181 & $l_{2}-l_{4}$ & 86 & $3 \ell_{2}-2 \ell_{3}-\pi_{3}$ & +256 & $\psi-\omega_{4}$ \\
\hline+ & 125 & $2 \ell_{2}-2 \ell_{4}$ & $-\quad 75$ & $3 \ell_{2}-4 \ell_{3}+\pi_{2}$ & +92 & $\omega_{2}-\omega_{3}$ \\
\hline+ & 14 & $3 l_{2}-3 l_{4}$ & +553 & $3 l_{2}-4 l_{3}+\pi_{3}$ & +156 & $\omega_{3}-\omega_{4}$ \\
\hline+ & 256 & $l_{2}-\pi_{1}$ & $+\quad 231$ & $3 l_{2}-4 l_{3}+\pi_{4}$ & & \\
\hline
\end{tabular}


Equatorial True Longitudes (Cont.)

\begin{tabular}{|c|c|c|c|c|c|}
\hline A & $a$ & A & $a$ & A & $\alpha$ \\
\hline \multicolumn{6}{|c|}{ THIRD SATELLITE } \\
\hline-19161 & $l_{2}-l_{3}$ & -83 & $\ell_{3}-\pi_{2}$ & +814 & $\ell_{2}-2 \ell_{3}+\pi_{4}$ \\
\hline$-\quad 303$ & $2 \ell_{2}-2 \ell_{3}$ & +48289 & $\ell_{3}-\pi_{3}$ & -3736 & G \\
\hline$-\quad 333$ & $3 l_{2}-3 l_{3}$ & $+\quad 47$ & $2 \ell_{3}-2 \pi_{3}$ & -114 & $2 \mathrm{G}$ \\
\hline$-\quad 19$ & $4 l_{2}-4 l_{3}$ & +20492 & $\ell_{3}-\pi_{4}$ & -331 & $5 G^{\prime}-2 G+48^{\circ} .6$ \\
\hline- & $5 \ell_{2}-5 l_{3}$ & $-\quad 217$ & $2 \ell_{2}-3 \ell_{3}+\pi_{3}$ & +192 & $\pi_{3}-\pi_{4}$ \\
\hline$-\quad 11$ & $6 l_{2}-6 l_{3}$ & $-\quad 97$ & $2 \ell_{2}-3 l_{3}+\pi_{4}$ & +122 & $\psi-\omega_{2}$ \\
\hline-1192 & $l_{3}-l_{4}$ & +150 & $l_{3}-2 \mathrm{G}-2 \Pi+\pi_{3}$ & -1867 & $\psi-\omega_{3}$ \\
\hline+4075 & $2 \ell_{3}-2 \ell_{4}$ & -1678 & $\ell_{3}-2 \ell_{4}+\pi_{4}$ & +167 & $\psi-\omega_{4}$ \\
\hline$+\quad 286$ & $3 \ell_{3}-3 \ell_{4}$ & -100 & $\ell_{2}-2 \ell_{3}+\pi_{1}$ & +267 & $\omega_{3}-\omega_{4}$ \\
\hline 67 & $4 \ell_{3}-4 l_{4}$ & -922 & $\ell_{2}-2 \ell_{3}+\pi_{2}$ & & \\
\hline$+\quad 19$ & $5 \ell_{3}-5 \ell_{4}$ & +1869 & $\ell_{2}-2 \ell_{3}+\pi_{3}$ & & \\
\hline \multicolumn{6}{|c|}{ FOURTH SATELLITE } \\
\hline$+\quad 161$ & $l_{1}-l_{4}$ & $-\quad 5775$ & $\ell_{4}-\pi_{3}$ & -8933 & G \\
\hline$+\quad 142$ & $2 \ell_{1}-2 \ell_{4}$ & +234697 & $l_{4}-\pi_{4}$ & -322 & $2 \mathrm{G}$ \\
\hline-636 & $l_{3}-l_{4}$ & +1078 & $2 \ell_{4}-2 \pi_{4}$ & +100 & $2 G^{\prime}-G+173^{\circ} .17$ \\
\hline$-\quad 319$ & $2 \ell_{3}-2 \ell_{4}$ & 278 & $\ell_{3}-2 \ell_{4}+\pi_{4}$ & -781 & $5 G^{\prime}-2 G+48^{\circ} \cdot 64$ \\
\hline-69 & $3 l_{3}-3 l_{4}$ & $+358 *$ & $2 \ell_{4}-2 G-2 I$ & +222 & $\psi-\omega_{3}$ \\
\hline 19 & $4 \ell_{3}-4 \ell_{4}$ & +1764 & $\ell_{4}-2 G-2 \Pi+\pi_{4}$ & -556 & $\psi-\omega_{4}$ \\
\hline
\end{tabular}

* $H$. Andoyer [Bull. Astr. 32, 1915] gives the corrected value +386 for this coefficient. 


\section{Radii Vectors}

(projected on the equator)

(in astronomical units of length)

\begin{tabular}{|c|c|}
\hline $\begin{aligned} r_{1} \times 10^{9}= & 2.81955\left[10^{6}+\right. \\
& 20 \cos \left(\ell_{1}-\ell_{2}\right) \\
- & 4125 \cos \left(2 l_{1}-2 \ell_{2}\right) \\
- & 20 \cos \left(3 \ell_{1}-3 l_{2}\right) \\
- & 15 \cos \left(4 \ell_{1}-4 \ell_{2}\right) \\
+ & 10 \cos \left(\ell_{1}-l_{3}\right) \\
- & 15 \cos \left(2 \ell_{1}-2 \ell_{3}\right) \\
- & 45 \cos \left(\ell_{1}-\pi_{1}\right) \\
- & 35 \cos \left(\ell_{4}-\pi_{3}\right) \\
- & \left.15 \cos \left(\ell_{1}-\pi_{4}\right)\right]\end{aligned}$ & $\begin{aligned} r_{2} \times 10^{9}= & 4.48612\left[10^{6}+\right. \\
& 55 \cos \left(\ell_{2}-l_{3}\right) \\
- & 9430 \cos \left(2 \ell_{2}-2 l_{3}\right) \\
- & 55 \cos \left(3 l_{2}-3 l_{3}\right) \\
- & 30 \cos \left(4 \ell_{2}-4 \ell_{3}\right) \\
- & 85 \cos \left(\ell_{2}-\pi_{2}\right) \\
- & 310 \cos \left(\ell_{2}-\pi_{3}\right) \\
- & \left.130 \cos \left(\ell_{2}-\pi_{4}\right)\right]\end{aligned}$ \\
\hline $\begin{aligned} r_{3} \times 10^{9}= & 7.15587\left[10^{6}+\right. \\
& 635 \cos \left(\ell_{2}-\ell_{3}\right) \\
+ & 15 \cos \left(2 \ell_{2}-2 \ell_{3}\right) \\
- & 15 \cos \left(3 \ell_{2}-3 l_{3}\right) \\
+ & 25 \cos \left(\ell_{3}-\ell_{4}\right) \\
& -140 \cos \left(2 l_{3}-2 \ell_{4}\right) \\
& -10 \cos \left(3 \ell_{3}-3 \ell_{4}\right) \\
& -1515 \cos \left(\ell_{3}-\pi_{3}\right) \\
& \left.-645 \cos \left(\ell_{3}-\pi_{4}\right)\right]\end{aligned}$ & $\begin{aligned} \mathbf{r}_{4} \times 10^{9}= & 12.58625\left[10^{6}+\right. \\
& 100 \cos \left(\ell_{3}-\ell_{4}\right) \\
& +20 \cos \left(2 \ell_{3}-2 \ell_{4}\right) \\
& +180 \cos \left(\ell_{4}-\pi_{3}\right) \\
& -7375 \cos \left(\ell_{4}-\pi_{4}\right) \\
& -25 \cos \left(2 \ell_{4}-2 \pi_{4}\right) \\
& \left.-55 \cos \left(\ell_{4}-2 G-2 \Pi+\pi_{4}\right)\right]\end{aligned}$ \\
\hline
\end{tabular}


Additional Terms of the Longitudes in Sampson's System

Let $\Delta v_{i}=2 \pi \times 10^{-8} \sum A \sin a(\mathrm{rad})$ be the correction to be added to $v_{i}$ in order to obtain the true longitude of $i^{\text {th }}$ satellite in Sampson's co-ordinate system.

\begin{tabular}{|c|c|c|}
\hline $\mathrm{i}$ & A & $a$ \\
\hline 1 & $\begin{array}{r}-11667 \\
-\quad 206 \\
-\quad 78\end{array}$ & $\begin{array}{l}2 \ell_{1}-2 \psi \\
2 \ell_{1}-\psi-\omega_{1} \\
2 \ell_{1}-\psi-\omega_{2}\end{array}$ \\
\hline 2 & $\begin{array}{rr}-11553 \\
-\quad 3514 \\
-\quad 186 \\
-\quad 44 \\
-\quad 267\end{array}$ & $\begin{array}{l}2 \ell_{2}-2 \psi \\
2 \ell_{2}-\psi-\omega_{2} \\
2 \ell_{2}-\psi-\omega_{3} \\
2 \ell_{2}-\psi-\omega_{4} \\
2 \ell_{2}-2 \omega_{2}\end{array}$ \\
\hline 3 & $\begin{array}{r}-11025 \\
+\quad 122 \\
-1303 \\
-\quad 264\end{array}$ & $\begin{array}{l}2 \ell_{3}-2 \psi \\
2 \ell_{3}-\psi-\omega_{2} \\
2 \ell_{3}-\psi-\omega_{3} \\
2 \ell_{3}-\psi-\omega_{4}\end{array}$ \\
\hline 4 & $\begin{array}{rr}-18661 \\
- & 1781 \\
+ & 189 \\
-\quad 83 \\
-\quad 28^{*}\end{array}$ & $\begin{array}{l}2 \ell_{4}-2 \psi \\
2 \ell_{4}-\psi-\omega_{4} \\
2 \ell_{4}-\psi-\omega_{3} \\
2 \ell_{4}-2 \omega_{4} \\
2 \ell_{4}-2 G-2 \pi\end{array}$ \\
\hline
\end{tabular}

* Only to be introduced if $\mathrm{H}$. Andoyer's correction in the equatorial longitude is adopted.

N.B. To obtain full-accuracy determinations of the positions, $v_{i}$ should be substituted to $l_{i}$ in the arguments $a$. 


\section{Latitude Series}

$b_{i}$ being the latitude of the $i^{\text {th }}$ satellite, relative to Sampson's $(x, y)-$ plane:

$$
\operatorname{tg} b_{i}=10^{-7} \Sigma B \sin \beta
$$

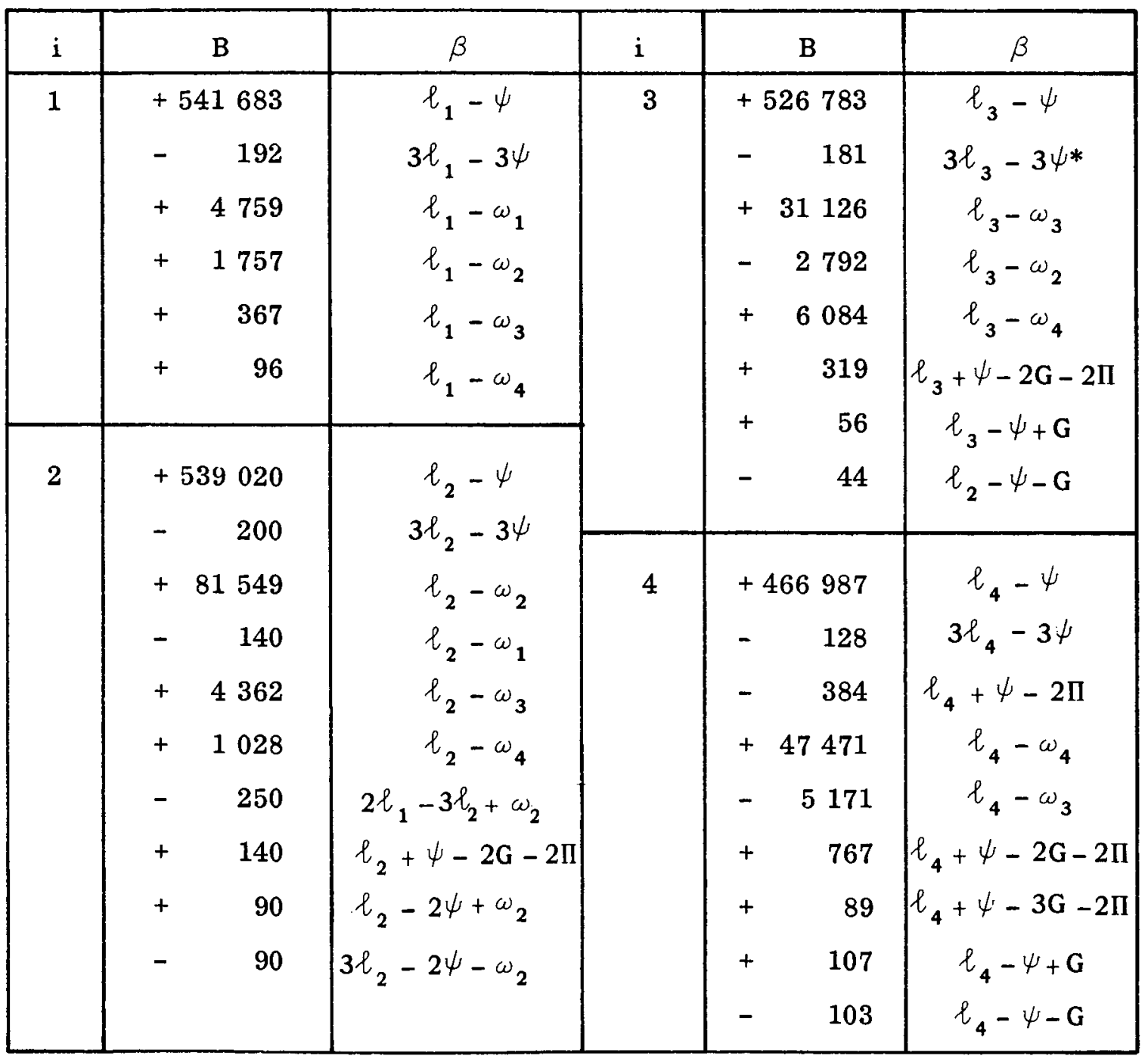

*Erroneously $3 \ell_{4}-3 \psi$ in Sampson's Tables. N.B. To obtain full accuracy determinations of the positions, $v_{i}$ should be substituted to $l_{i}$, in
the arguments $\beta$ ! 


\section{BIBLIOGRAPHY}

1. Battin, Richard H., "Astronautical Guidance," McGraw-Hill Book Company, 1964.

2. Escobal, Pedro R., "Methods of Orbit Determination," John Wiley and Sons, 1965.

3. Nelson, Walter C., Loft, Ernest E., "Space Mechanics" Prentice Hall, Inc., 1962.

4. Danby, J. M. A., "Fundamentals of Celestial Mechanics," MacMillan Company, 1964.

5. Baker, R. M. L.; Makemson, M. W., "An Introduction to Astrodynamics," Academic Press, 1960.

6. Battin, R. H., "The Determination of Round-Trip Planetary Reconnaissance Trajectories," J. Aerospace Science, Volume 26, pp. 545-567, September, 1959.

7. "Programmer's Manual for Quick Look Mission Analysis Program," Philco, WDL Division, WDL-TR2217, Contract NAS 5-3342, January 1964.

8. Kaufman, B; Newman, C. R., Chromey, F., "Gravity Assist Optimization Technique Applicable to a Variety of Space Missions," GSFC Document X-507-66-373, August 1966.

9. Newman, C. R.; Kaufman, B., "The Effects of Jovian Encounter Geometry on the Post-Encounter Heliocentric Trajectory." GSFC Document to be published.

10. Minovitch, M. A., "The Determination and Characteristics of Ballistic Interplanetary Trajectories Under the Influence of Multiple Planetary Attractions," JPL Technical Report No. 32-464, October 31, 1963. 
11. Minovitch, M. A., "Utilizing Large Planetary Perturbations for the Design of Deep-Space, Solar Probe, and Out-of-Ecliptic Trajectories," JPL Technical Report No. 32-849, December 15, 1965.

12. Porter, R. F., Luce, R. G.; Edgecombe, D. S., "Gravity-Assisted Trajectories for Unmanned Space Exploration," Battelle Memorial Institute Report No. BM-NLVP-FTR-65-1, September 23, 1965.

13. Niehoff, John C., "An Analysis of Gravity Assisted Trajectories to Solar System Targets," IIT Research Institute, AIAA Paper No. 66-10, January 1966.

14. Vonbun, F. O., "Transfer Geometry, Communications Conditions and Definitions for Galactic Probe Analysis," Mission Analysis Office Technical Study, Goddard Space Flight Center, October 4, 1965.

15. Sturms, F. M., Jr., "Trajectory Analysis of an Earth-Venus-Mercury Mission in 1973," JPL Technical Report No. 32-1062, January 1, 1967.

16. Herget, Paul, "A Device in Satellite Perturbation Computations", Astronomical Journal, No. 1162 pp. 177-178, 1946.

17. Linnekin, Jerry S., Belliveau, L. J., "FNOL 2, A Fortran (IBM 7090) Subroutine for the Solution of Ordinary Differential Equations with Automatic Adjustment of the Interval of Integration," U.S. Naval Ordinance Laboratory, NOLTR 63-171, July 17, 1963.

18. "Wanderers in the Sky," Edited by Thornton Page and Lou Williams Page, Macmillan Co., N. Y., 1965.

19. Baker, Robert H., "Astronomy," D. Van Nostrand Co, Inc. 1964.

20. "Space Facts" Compiled and published by the General Electric Missile and Space Vehicle Department, 1960.

21. Michaux, C. M., "Handbook of the Physical Properties of the Planet Jupiter," Douglas Aircraft Company, NASA contract publication, NASA SP-3031, 1967.

22. Porter, J. C., "The satellites of the Planets," J. Brit. Astron. Assoc., Vol. 70, No. 1, January 1931.

23. Sampson, R. A., "Tables of the Four Great Satellites of Jupiter," University of Durham Observatory, William Wesley and Son, 1910. 
24. Sampson, R. A., "Theory of the Four Great Satellites of Jupiter," Publication of the Royal Astronomical Society, Vol. LXIII, 1920.

25. De Sitter, W., "Jupiter's Galilean Satellites," Monthly Notices Roy. Astron, Soc., Vol. 91, No. 7, May 1931.

26. "Explanatory Supplement to the Ephemeris," Prepared Jointly by the Nautical Almanac Offices of the United Kingdom and the U.S.; Her Majesty's Stationary Office, 1961.

27. Andoyer, H., "Sur le Calcul des Ephe'merides des Quatre Anciens Satellites de Jupiter," Bulletin Astronomique, 32, 177, 1915.

28. Mello, Ferraz S., Bull. Astr., (3), Vol. 1, fasc. 4, 1966.

29. Marsden, B. G., "The Motion of the Galilean Satellites of Jupiter," Dissertation, Yale University, 1966.

30. Private Communication with Dr. Jean L. Sagnier, Bureau des Longitudes, Palais de L'Institute, Paris, France.

31. Coady, Robert E., "Trajectory Analysis - Near-Earth and Jupiter-Approach Phases of the 1972 Galactic Jupiter Probe Mission," Mission Analysis Office Technical Study, Goddard Space Flight Center, April 28, 1967. 